\title{
Tunnelling in rare-earth nitride structures
}

\author{
by \\ Jackson Miller
}

\author{
A thesis \\ submitted to the Victoria University of Wellington \\ in partial fulfilment of the \\ requirements for the degree of \\ Master of Science \\ in Physics.
}

Victoria University of Wellington

2018 



\begin{abstract}
GdN thin film device structures, including magnetic tunnel junctions (MTJs), were grown by physical vapour deposition and their electrical properties were investigated. Growth compatibility between $\mathrm{GdN}$ and various contact metals ( $\mathrm{Al}, \mathrm{Au}, \mathrm{Gd}$ and $\mathrm{Nb}$ ) was assured using X-ray diffraction (XRD) and scanning electron microscopy (SEM) techniques. I developed a photomask and lithographic process to isolate electrical behaviour perpendicular to the plane of the films. $\mathrm{Al}$ and $\mathrm{Au}$ were confirmed to make ohmic contact to GdN, while Gd and $\mathrm{Nb}$ both formed Schottky-like barriers at the interface with GdN. In MTJ structures, device electrical characteristics were dominated by tunnelling behaviour through the GaN barrier layer. The Simmons model was successfully applied to tunnelling measurements of $\mathrm{Al} / \mathrm{GdN} / \mathrm{GaN} / \mathrm{GdN} / \mathrm{Gd}$ structured MTJs to determine the barrier properties. MTJs grown with Al bottom contacts were grown with $1.5 \mathrm{eV}$ potential barrier height and $2.5 \mathrm{~nm}$ width. Finally, MTJs contacted with $\mathrm{Nb}$ exhibited a large magnetoresistance $(>500 \%)$, greater than GdN-based MTJs recorded in the literature [Warring et al. "Magnetic Tunnel Junctions Incorporating a Near-Zero-Moment Ferromagnetic Semiconductor", Phys. Rev. Appl., vol.6, p.044002, 2016].
\end{abstract}




\title{
Acknowledgments
}

\author{
Ehara taku toa, he takitahi, he toa takitini - Whakatauki
}

I do not believe anyone truly accomplishes anything on their own, and a thesis is certainly not the kind of document that would disabuse me of this notion.

To whānau first, thank you for being mine. Suzanne and Jim, Finn and Stella you contributed more to this over 24 years than I did over 2, probably. Wider whānau, thank you also. The previous statement is no less true for you, but for proximity. Thank you especially to Derek, Susan, Ione, Sophie and particularly Judy. You don't choose your family, but you do choose whether or not to accept them. I am forever grateful.

To all my friends, present and past, you are a major part of my life and I cherish you. You made me.

To Tatsushi and Fabio, thanks for the food. (I have been planning these acknowledgements too long for the two of you not to appear).

The majority of the thanks for the document you are holding go to Franck Natali, Joe Trodahl and Ben Ruck. This project would be nothing without you, and I look forward to future projects and learning. Will, Felicia, Jay, Bart, Harry and Ali; you are fantastic to work with. Extra thanks to Fergus; you contributed the most, at just the right moment.

To the wider Cleanroom group, the Raman group and in particular Leo and Matthias; thanks for being the work bros.

To Uli, Michele, Natalie and the other academics; thanks for the discussion. 
Most importantly, Ellie Gill, I adore you. 


\section{Contents}

1 Introduction 1

1.1 Spintronics ........................ 1

1.2 Applications .................... 2

1.2.1 Magnetic Tunnel Junctions (MTJs) . . . . . . . . . . . 3

1.2.2 Next Generation Devices: A Materials Science Problem! . . . . . . . . . . . . . . 6

1.3 Rare-Earth Nitrides (RENs) . . . . . . . . . . . . 7

1.4 Motivation and Context of This Work . . . . . . . . . . 9

1.5 Outline ....................... 10

2 Film Growth and Characterisation $\quad 13$

2.1 Film Fabrication . . . . . . . . . . . . . . . . . . . 13

2.2 Electrical Characterisation . . . . . . . . . . . . . 15

2.3 Physical Characterisation . . . . . . . . . . . 16

2.4 Developments in Fabrication . . . . . . . . . . . . . 24

2.4.1 Optical Lithography . . . . . . . . . . . . . 27

2.5 Interpretation of Electrical Measurements . . . . . . . . . 30

2.5.1 Schottky Contacts . . . . . . . . . . . . . 33

2.5.2 The Simmons Model . . . . . . . . . . . . . 35

3 GdN structures with Metal Contacts $\quad 39$

$3.1 \mathrm{GdN}$ with $\mathrm{Al}$ contacts . . . . . . . . . . . . . . . . . 40

3.2 GdN with Gd contacts . . . . . . . . . . . . . . . . 44 
3.3 MTJs with metal contacts . . . . . . . . . . . . . 47

3.4 Discussion . . . . . . . . . . . . . . . . . . . . 49

4 GdN Structures with Superconducting Contacts 53

$4.1 \mathrm{GdN}$ with $\mathrm{Nb}$ contacts . . . . . . . . . . . . . . . 53

4.2 MTJs with $\mathrm{Nb}$ contacts . . . . . . . . . . . . 60

4.3 Discussion ...................... 63

5 Conclusions $\quad 67$ 


\section{Chapter 1}

\section{Introduction}

\subsection{Spintronics}

Following the invention of the transistor, the latter half of the 20th century saw a revolution associated with the development of semiconductor materials, in particular, silicon. The relentless development of silicon microelectronics over this period is often summarised by reference to 'Moore's Law', the prediction that the number of transistors on a single computer chip (a strong indicator of the processing power of the electronics) would double every eighteen months ${ }^{1}$. While the predictive power of Moore's Law has stood up to scrutiny for decades, nanofabrication techniques are encroaching on fundamental size limits and constraints imposed by the uncertainty principle[2]. As early as the 1990s, technologies based on different physical principles were roadmapped as an alternative to silicon. One such alternative was 'spintronics'2.

\footnotetext{
${ }^{1}$ Moore's original 1965 paper actually described an annual doubling of the number of components per integrated circuit, revised in 1975 to a biannual doubling. The prediction of doubling of processing power every eighteen months can more accurately be attributed to Intel executive David House (this estimate is based not only on the number of transistors per integrated circuit but also increased clock speeds.).[1]

${ }^{2}$ A portmanteau of SPIN TRansport electrONICS first used (in 1993) to designate a research program initiated by the US Defense Advanced Research Projects Agency
} 
Silicon electronics, one of the world's major industries, leverages the existence and absence of electric charge into a binary system of information controlled by the manipulation and transport of electrons. Spintronics takes advantage, not only of the charge of the electrons, but also the property of electron $\operatorname{spin}^{3}$. In contrast to the widespread commercial application of charge-based electronics, exploitation of the spin of the electron did not become commonplace in a technological context until the 2000s[3]. This paradigm shift within electronics research towards applications of magnetic materials (which harness the spin of the electron) has since lead to undisputed technological impact (in digital memory and magnetic sensors) and fundamental research[6].

\subsection{Applications}

The applications of spintronics research are already seen in commercially available devices, such as read heads of hard disk drives and magnetic field sensors. Most of these commercial technologies stem from the giant magnetoresistance (GMR) effect discovered in multilayered thin films in 1988[7] $]^{4}$. These films comprised of alternating layers of ferromagnetic (iron) and non-magnetic (chromium) metals, where the thickness of the non-magnetic $\mathrm{Cr}$ layers was used to control the exchange interaction between the ferromagnetic layers. This resulted in spin-dependent scattering of electrons, causing a large magnetoresistance ratio (called "giant" to distinguish the effect from anisotropic magnetoresistance). The magne-

\footnotetext{
(DARPA). [3]

${ }^{3}$ The spin of an electron describes its intrinsic angular momentum (that not resulting from its orbital movement) and is widely held to be a purely quantum phenomenon (see [4] and [5] for discussion).

${ }^{4}$ For the discovery of the GMR effect, Albert Fert and Peter Grünberg were presented the 2007 Nobel prize in physics[8].
} 
toresistance ratio is defined as

$$
M R=\frac{R_{A P}-R_{P}}{R_{P}}=\frac{\Delta R}{R_{P}}
$$

where $R_{P}$ is the electric resistance of the multilayer when the magnetisation directions of the ferromagnets are aligned parallel with one another (usually in the presence of an externally applied magnetic field). Typically $R_{A P}$ is the electric resistance of the multilayer when the magnetisation directions of the ferromagnets are aligned antiparallel, in polycrystalline films (such as the ones in this study) the random orientation of magnetic domains is taken to provide the high resistance state equivalent to antiparallel alignment.

The GMR effect is observed in magnetic multilayered systems whether the current is applied in the plane of the film (CIP) or perpendicular to the plane $(\mathrm{CPP})^{5}$. Because the GMR effect is stronger in the CPP geometry[6] most applications favour this orientation.

\subsubsection{Magnetic Tunnel Junctions (MTJs)}

The commercial success of GMR-based devices (such as the spin valves used in read heads) has motivated the development of new devices that leverage the spin of the electron, such as magnetic tunnel junctions (MTJs). While the magnetoresistance ratios of spin valves are large relative to anisotropic magnetoresistance effects, much larger ratios are possible if one exploits phenomena such as electron tunnelling. One example of this is provided by the MTJ, a trilayer structure consisting of two ferromagnetic layers (electrodes 1 and 2 ) seperated by a thin $(<5 \mathrm{~nm}$ ) insulating layer (see Figure 1.1). This insulating layer inhibits conduction through the device, but allows the passage of a tunnelling current. The use of strongly spinpolarised ferromagnets in the MTJ causes the tunnelling current to depend

\footnotetext{
${ }^{5}$ Theoretical description of the GMR effect in CIP and CPP geometries is provided in references [9] and [10] respectively.
} 
on the magnetisation of the electrodes (explained below), and this leads to magnetoresistance ratios larger than those seen in commercially available spin valves.

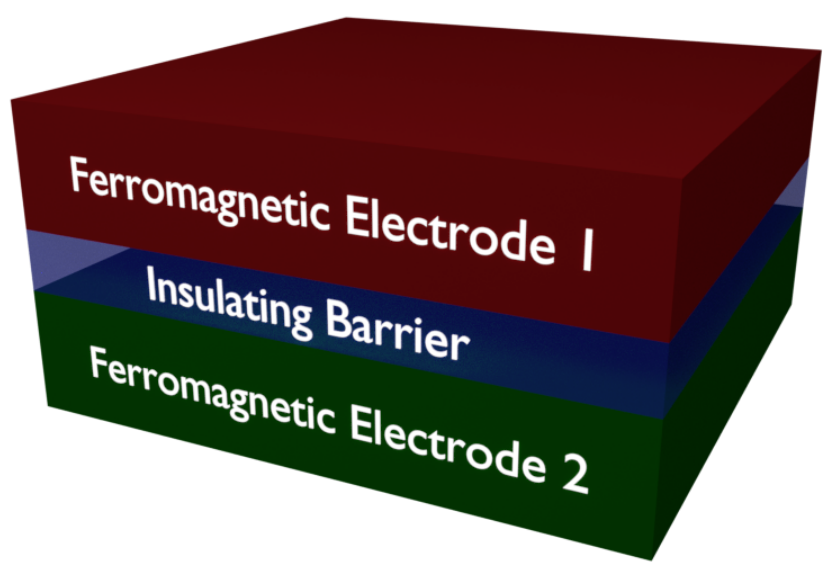

Figure 1.1: A schematic of the MTJ structure.

The high magnetoresistance in these devices is a direct consequence of ferromagnetic exchange splitting in the electrodes, an effect produced by manipulating an externally-applied magnetic field to produce a high or low tunnelling current, as desired. Many models describe the general problem of electron tunnelling (that of Simmons is implemented in later chapters[11, 12]). In general, these models describe an electron vacating a state in electrode 1 and moving to occupy a state of the same spin in electrode 2 (see Figure 1.2). In the case of an MTJ the exchange splitting moves the bands up or down in energy depending on the direction of the electron spin. If the electrodes are magnetised along the same axis (parallel), this effect does not significantly change the magnitude of the tunnelling current. However, if the magnetisations are forced into an antiparallel configuration, the "up" spin direction is no longer the same for both electrodes. Electrode 2 has few vacant states available to tunnel into (as occupied states are prohibited by Pauli exclusion). This frustrates the tunnelling current, returning a high resistance. The change in the resis- 
tance as a function of the magnetisation alignment in the device electrodes provides a large magnetoresistive effect.

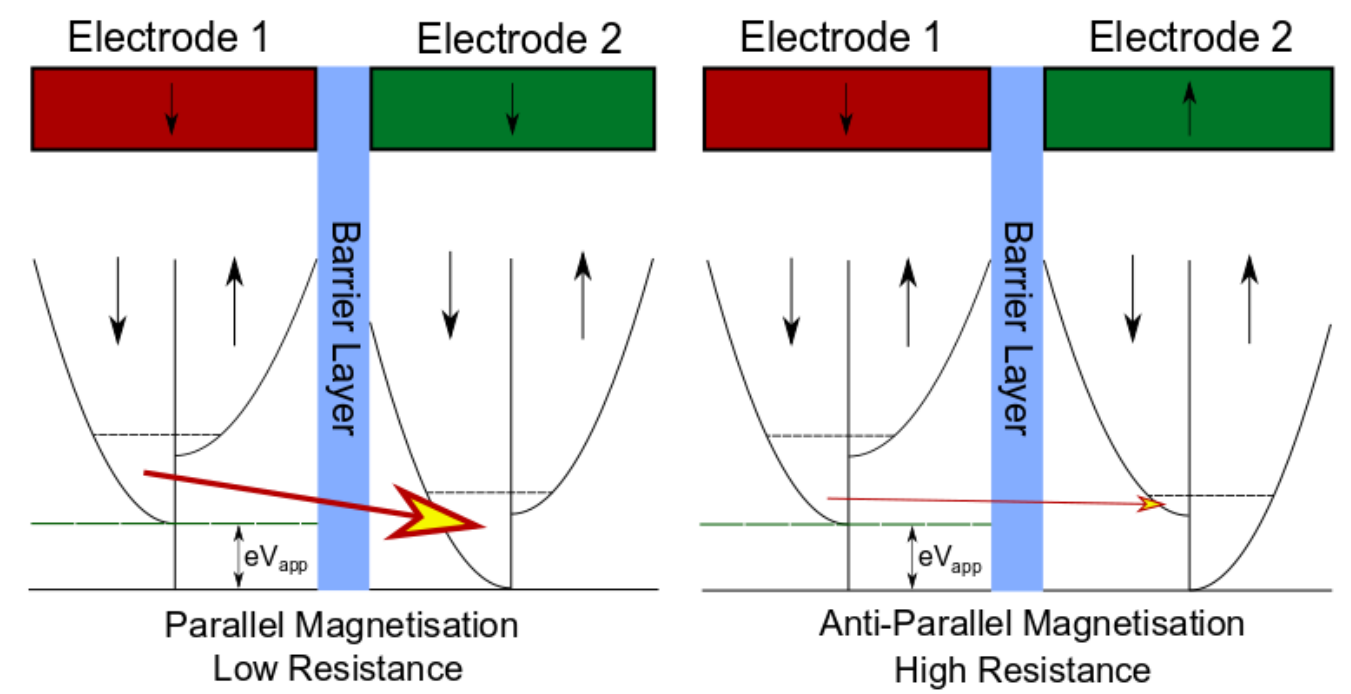

Figure 1.2: Schematic diagram of the MTJ electrode conduction bands in parallel and antiparallel configurations. These configurations correspond to the low and high resistance states respectively. The conduction band of Electrode 1 is displaced to higher energy by $e V_{a p p}$ when the device is operating, where $V_{a p p}$ is the applied voltage and $e$ is the electronic charge.

The earliest theoretical description of MTJs was made by Julliére[13], who suggested a model relating the magnetoresistance ratio, $M R$, to the spin-polarisation of the device electrodes:

$$
M R=\frac{2 P_{1} P_{2}}{1-P_{1} P_{2}}
$$

$P_{1}$ and $P_{2}$ refer to the spin-polarisation in electrode 1 and 2 respectively ${ }^{6}$. The Julliére model fails to explain much of the experimental data within the field, largely due to its simplicity (see [14] for review). Nonetheless, Julliére did identify the major role played by electrode spin-polarisations

\footnotetext{
${ }^{6}$ The equation as presented does not appear in the Julliére paper[13], but is an equivalent formulation in terms of the resistance rather than conductance (see [6])
} 
in determining the magnetoresistance of the device. The strong dependence described by the model implies the possibility of extremely high magnetoresistance in devices with semiconducting ferromagnetic electrodes, as ferromagnetic semiconductors may be engineered to have near $100 \%$ spin polarisation.

\subsubsection{Next Generation Devices: A Materials Science Prob- lem!}

It is here that one finds a gaping hole in the spintronics portfolio: the dearth of materials with both magnetic and semiconducting properties has greatly impeded progress towards semiconductor-based spintronics. Existing commercial technologies rely on metallic ferromagnets as sources of spin injection and detection, while transport, manipulation and storage occur in non-magnetic layers. The impedance mismatch between metallic ferromagnets and non-magnetic semiconductors inhibits efficient injection and detection of spin, limiting the development of spintronics device technologies. The combination of ferromagnetic and semiconducting characteristics in a single material would combat this roadblock to robust semiconductor spintronics.

The scarcity of intrinsic ferromagnetic semiconductors has led to attempts to magnetically dope existing non-magnetic semiconductor systems, such as GaAs and GaN[15, 16]. These doped materials are classed as dilute magnetic semiconductors (DMS) and were initially expected to allow the operation of spintronic devices at room temperature[17]. Although a lot of progress has been made in DMS research over the last ten years, the ferromagnetic exchange interaction in these materials is still not completely understood. The segregation of secondary phases, and the generation of defects when increasing the concentration of dopant atoms, remain a problem for the growth of these materials. Furthermore, both the electrical conduction and magnetic behaviour of such systems are affected 
by the introduction of the dopant atoms. The lack of independent control of these properties constitutes a distinct disadvantage from the perspective of device functionality $[18,19]$.

A solution to the poverty of ferromagnetic semiconductors is found in the rare-earth nitride (REN) series of compounds. Aerts et al. [20] predicted in the early 2000s that the REN series was likely to contain halfmetallic compounds, generating significant research interest. This was compounded by the claim that some of the RENs would have highly spinpolarised mobile electrons. Experiments in the following decade contradicted these predictions, many members of the REN series being intrinsic ferromagnetic semiconductors[21]. The coexistence of ferromagnetism and semiconductivity makes the RENs very attractive for spintronics applications.

\subsection{Rare-Earth Nitrides (RENs)}

The rare-earth nitrides (compounds of the form LN with L a Lanthanide) were first studied well in advance of the Aerts prediction, with research being undertaken on bulk samples in the 1960s and 70s[22, 23]. However, poor stoichiometry and rapid oxidation of the samples in air led to the discontinuation of this research. Advances in fabrication technology have since allowed the resumption of experimental research, with fabrication of the RENs most commonly achieved under ultra-high vacuum (UHV) using Molecular Beam Epitaxy (MBE)[24] or Pulsed Laser Deposition (PLD) [25] $]^{7}$.

The RENs crystallise in the rocksalt structure (two interleaved FCC lattices, or equivalently, an FCC lattice with a two-atom basis. See Figure 1.3) with lattice parameters ranging from $5.305 \AA$ for $\mathrm{LuN}$, down to $4.76 \AA$ for LaN. Vacancies at the nitrogen lattice sites provide an intrinsic

\footnotetext{
${ }^{7}$ More recently, growth has been achieved using Chemical Vapour Deposition (CVD)[26]
} 


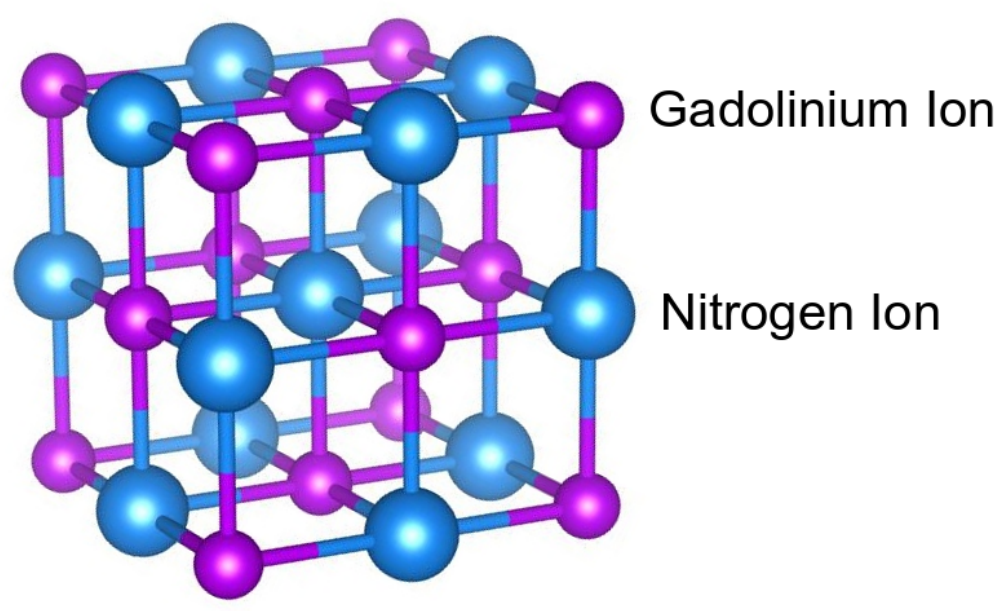

Figure 1.3: Image of the rocksalt structure of the RENs, showing the $\mathrm{Gd}^{3+}$ ions in purple and the $\mathrm{N}^{3-}$ ions in blue.

n-type doping[21] to many members of the series. Each empty nitrogen site contributes three electrons to the lattice, of which at least one is unbound. The electrical conduction of the RENs results from a $5 \mathrm{~d}$ conduction band, while the strong magnetic character of the materials comes from the partially-filled $4 \mathrm{f}$ shell. The strongly-bound $4 \mathrm{f}$ electrons contribute little to the electrical conduction, and this allows control of the materials' electrical properties independent of their magnetic character (this has been achieved most successfully by magnesium doping in GdN[27]). The independent control of electrical and magnetic properties seen in the RENs cannot currently be achieved in dilute magnetic semiconductors and this point of difference gives the RENs huge scope for applications in the world of spintronics.

Because the occupation of the $4 \mathrm{f}$ electron states of the rare-earth ions is the driver of magnetism in the RENs, there is significant variation in magnetic behaviour across the series. Gadolinium nitride $(\mathrm{GdN})$ is the member of the series for which the $4 \mathrm{f}$ shell is half-filled, its ground-state orbital angular momentum is completely balanced by the occupation of all the $4 \mathrm{f}$ 
suborbitals and contributes nothing to the total angular momentum. The magnetic moment is thus completely dominated by the spin-moment of the $4 \mathrm{f}$ electrons. Below $50 \mathrm{~K} \mathrm{GdN}$ is ferromagnetically ordered with magnetic polarons providing an increase in the Curie temperature (up to 70K) in heavily n-doped samples[28]. The magnetisation saturates at $7 \mathrm{Bohr}$ magnetons per gadolinium ion and the coercive field is $\sim 0.02 \mathrm{~T}[25,29]$.

The coincidence of ferromagnetic behaviour with the capacity for tuning the electrical conductance in one material ensures that the RENs are expediently placed to offer utility in spintronics applications. GdN has already found use in such practical devices as spin filters[30], field-effect transistors[31], and as barriers in tunnelling structures[32]. Proof-of-concept MTJs using GdN and SmN electrodes with non-magnetic barriers have been realised[33], and the VUW spintronics group has provisional patents related to the development of this technology[34, 35]. This thesis explores the integration of GdN-based MTJs with niobium $(\mathrm{Nb})$ and other metals.

\subsection{Motivation and Context of This Work}

Increasing demand for centralised computation facilities, driven by cloud computing, support of mobile devices and computation-intensive applications has triggered a move towards large-scale supercomputer mainframes and data centres in recent times[36]. But our requirement for ever greater computational power and storage has risen to insatiable levels and centralised facilities are now estimated to account for $7 \%$ of the world's total energy budget with projections to $13 \%$ by $2030[37,38]$. During the era of microelectronics, growing demand for computational power was offset by the exponential growth in processing power described by Moore's Law, yet the fundamental constraints on conventional semiconductor electronics mean that Complementary Metal-Oxide Semiconductor (CMOS) technology (upon which much of today's high performance computing is based) may not be able to increase efficiency rapidly enough to forestall 
this issue[2, 36].

Highly efficient computation already exists in the form of superconducting computing (using what is called Rapid Single Flux Quantum (RSFQ) technology)[36]. RSFQ circuits are based on Josephson junctions, superconducting devices that switch quickly ( $\sim 1$ ps) and with little energy dissipation $\left(<10^{-19} \mathrm{~J}\right)$. The use of superconducting elements as the basis of this technology places strong requirements on the cooling system (all operation at $\sim 5 \mathrm{~K}$ for circuits using $\mathrm{Nb}$, a typical superconductor used for this purpose). However, even including cooling requirements RSFQ circuits require no more power than conventional supercomputing technology, so refrigeration is no barrier to higher energy efficiency[36].

Hypres Inc. is one company producing commercially available superconducting computation products[39]. What is missing in attempts by companies such as Hypres Inc. to develop competitive superconducting computing are memory elements compatible with RSFQ technology[36]. Present interest is centred around MTJs and their potential as a non-volatile magnetic random access memory (MRAM) element[40]. The properties of the RENs make them an attractive option in the development of these memory elements and for this reason the spintronics group at VUW have entered into a collaboration with Hypres Inc. The integration of RENbased MTJs with $\mathrm{Nb}$ is the primary goal of this project. To this end, Hypres provided substrates templated with $\mathrm{Nb}$ to establish growth compatibility with the RENs. $\mathrm{Nb}$ was grown in house for the subsequent electrical measurements as lithographic patterning was required.

\subsection{Outline}

The remainder of this thesis is structured thus:

- Chapter 2 details the fabrication and characterisation of the device structures grown for this project. It documents the development of 
lithographic techniques and models used to interpret electrical measurements.

- Chapter 3 looks at results taken from structures with metal contacts. This includes information that can be drawn from the data about contacting $\mathrm{GdN}$ and successful modelling of the electrical behaviour of GdN-based MTJs.

- Chapter 4 presents the integration of $\mathrm{GdN}$ with $\mathrm{Nb}$ (from an electrical perspective) and the presentation of strong tunnelling magnetoresistance in GdN-based MTJs grown on $\mathrm{Nb}$. 


\section{Chapter 2}

\section{Film Growth and Characterisation}

This thesis is primarily focussed on the electrical characterisation of rareearth nitride-based thin film devices. Physical methods were also used to assess thin film quality and thickness.

\subsection{Film Fabrication}

The films and devices used in this study were grown by physical vapour deposition, using an electron beam to evaporate bulk materials under ultrahigh vacuum (UHV). The UHV system consists of a main growth chamber pumped using a high throughput turbomolecular pump and a load-lock chamber pumped out using a smaller turbopump. Substrates are loaded into the growth chamber via the load-lock chamber using a magnetically coupled transfer arm. A system of clips on the arm and the growth chamber manipulator interface with grooves on the substrate holder to hold the sample. Rotation of the system manipulator moves the sample between the transfer arm and the manipulator itself. By venting only the loadlock chamber when transferring substrates in and out of the system, and waiting for the load-lock to approach the vacuum pressure of the growth chamber before moving samples between chambers, the base pressure of the main chamber is kept below $10^{-8}$ mbar at all times. 
Pressures inside the growth chamber are monitored during thin film deposition using a cold cathode gauge. The residual gases in the UHV chamber are analysed using a Pfeiffer quadrupole mass spectrometer before and after the growth of the films. Nitrogen gas forms the majority of the residual gas makeup, with water vapour kept below $10^{-9}$ mbar.

The growth chamber contains an electron gun with holders for up to four source materials ( $\mathrm{Ga}, \mathrm{Al}, \mathrm{Gd}$ and $\mathrm{Nb}$ ), and a small crucible used for the evaporation of $\mathrm{Sm}$ by resistive heating. The deposition rate is monitored in-situ by a quartz crystal microbalance to ensure films are grown to consistent thicknesses. This system is used in tandem with ex-situ measurement techniques, such as electron microscopy and X-ray reflectivity measurements to assess the physical characteristics of the films.

The films were deposited on thermally oxidised silicon wafers, where a 300nm layer of amorphous $\mathrm{SiO}_{2}$ on top of $\mathrm{Si}$ was used as the growth surface. The substrate was unheated during growth, ensuring the polycrystallinity of the deposited films. Nitrogen gas was introduced to the chamber up to a pressure of $10^{-4}$ mbar to react with the evaporated rareearth metal forming the REN thin films. Metal contacts of $\mathrm{Al}, \mathrm{Gd}$ and $\mathrm{Nb}$ were grown with nominally no nitrogen flow rate in order to achieve low pressures in-situ and high purity metallic films. One of the features of the rare-earth elements that makes for ease of growth is the catalytic breakdown of molecular nitrogen that occurs on the growing surface. This allows the growth to be carried out under pure $N_{2}$ and renders the use of ammonia as a precursor, or the ionisation of molecular nitrogen, unnecessary $^{1}$.

The RENs were found in the 1960s and 70s to react strongly with oxygen. This poses a problem for the characterisation of the RENs as the films rapidly decompose when removed from vacuum after growth. To combat this, a passivating layer (often referenced as a capping layer or cap) is grown over the REN film to inhibit the oxidation. Because this study fo-

\footnotetext{
${ }^{1}$ these are necessary for growth of other nitride compounds, such as AlN and GaN
} 
cused on measurements of devices where the current runs perpendicular to the plane of the film, it was necessary to cap the samples using metals (rather than the customary GaN or AlN layer used for optical measurements or in-plane resistivity). This serves a second purpose, as the metal capping layer functions as an electrical contact for device characterisation.

\subsection{Electrical Characterisation}

Electrical measurements were made using a Quantum Design Physical Property Measurement System (PPMS) in cases that the electrical response to an applied magnetic field was of interest, or when the required temperature was very low (The PPMS can access temperatures as low as $2 \mathrm{~K}$ ). Other electrical measurements were obtained using a helium closed-cycle cryostat with a minimum temperature of $4 \mathrm{~K}$.

\section{PPMS}

The PPMS system contains a sample stage mounted on a rotator rod within a superconducting magnet that can provide magnetic fields up to 9 Tesla. Electrical transport measurements can be performed for any temperatures within a $2-300 \mathrm{~K}$ range. The sample is thermally anchored using lowtemperature GE varnish, and electrical contacts are applied to the sample using silver paint. The opposite ends of these wires are soldered to gold pads on the sample stage which houses interconnects and gold pins that interface with the PPMS computer and allow the PPMS software to control and $\log$ sample data.

\section{Closed Cycle Cryostat}

The closed-cycle cryostat is used for electrical measurements for which a magnetic field is not required. The system is kept under vacuum to prevent heat flow by conduction and convection. An inner radiation shroud 
keeps the sample protected from room temperature radiation from outside the cryostat. The samples are mounted on a copper hearth and thermally anchored using Apiezon vacuum grease. Silver paint is used to make contact to the films using copper wire. The far end of the wire is soldered to electrical posts that are connected via an electrical feedthrough to the current source unit and digital volt/current meters.

\subsection{Physical Characterisation}

The thin films were characterised by means such as X-Ray Diffraction (XRD), X-Ray Reflectivity (XRR), Atomic Force Microscopy (AFM) and Scanning Electron Microscopy (SEM) to identify crystalline phases, confirm thicknesses and profile edge, surface and interface quality.

\section{X-Ray Diffraction}

X-Ray Diffraction (XRD) identifies crystal phases and film texturing ${ }^{2}$. It is imperative for reproducibility of device structures that these properties are consistent across film growths. A PANalytical "X'pert Pro" diffraction instrument was used to assess these qualities, with incident $K_{\alpha}$ radiation provided by a copper $(\mathrm{Cu})$ target $^{3}$. Figure 2.1 shows the geometry used to measure $x$-ray scattering. Peaks detected at the measurement angle $2 \theta$ are interpreted using the Bragg diffraction condition

$$
d=\frac{n \lambda}{2 \sin \theta}
$$

to provide information about the texturing and crystal structure normal to the plane of the film (here, $d$ is the distance between crystal planes, $n$ is an integer, $\lambda$ is the wavelength of the incident $\mathrm{x}$-rays and $\theta$ is the scattering angle, half the measured peak angle $2 \theta$ )[41].

\footnotetext{
${ }^{2}$ Film texturing references the preferential growth of a particular crystal orientation normal to the plane of the film.

${ }^{3}$ The $K_{\alpha}$ wavelength associated with $\mathrm{Cu}$ is $1.54 \AA$
} 


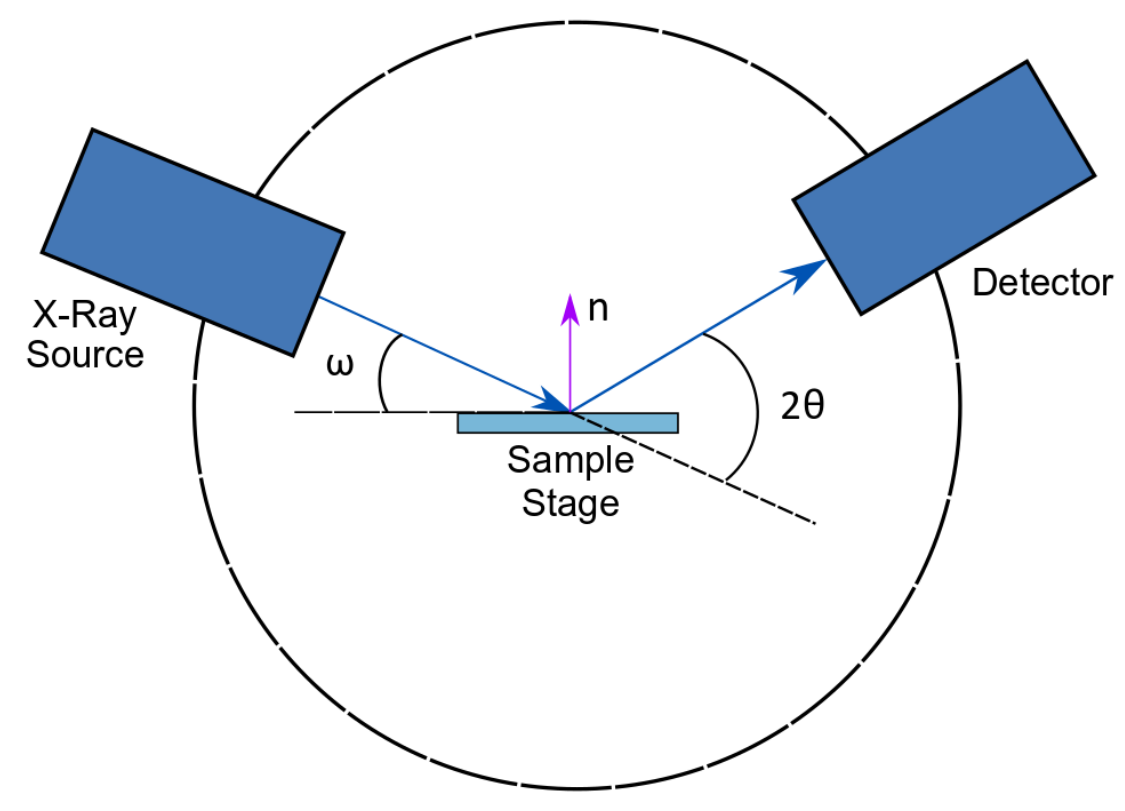

Figure 2.1: Schematic of the X-Ray Diffraction geometry showing the incident angle $\omega$ and the scattering angle $2 \theta$. Measuring the scattering angle in this geometry allows inference of crystal properties in the direction normal to the film plane (labelled n).

The lattice parameter of GdN was derived from the crystal spacing, $d$, for all samples, giving a mean value of $a=5.01 \AA$ and a standard deviation of $0.04 \AA$. Deposited at ambient temperature GdN grows polycrystalline phases (See Figure 2.2), so all crystal orientations (relative to the growth direction) are present in the sample. The absence of [100], [210],[211] etc. peaks (those peaks [h $\mathrm{k} \mathrm{l}$ ] for which $\mathrm{h}, \mathrm{k}$ and $\mathrm{l}$ are not all even or all odd) in the XRD measurements indicates an FCC crystal structure and the relative intensity of the peaks shows strong texturing in the [111] direction (preference for the crystal [111] direction to grow normal to the film plane). For FCC crystals ${ }^{4}$ the relationship between the lattice

\footnotetext{
${ }^{4}$ Actually any cubic crystal with $\mathrm{h}, \mathrm{k}$ and $\mathrm{l}$ pointing along the orthogonal unit cell directions.
} 
parameter and the distance between the lattice planes is given by

$$
a=d \sqrt{h^{2}+k^{2}+l^{2}}
$$

where $h, k$ and $l$ give the orientation of the measured peak $^{5}$. The values recorded in this project agree with those quoted in the literature[21].

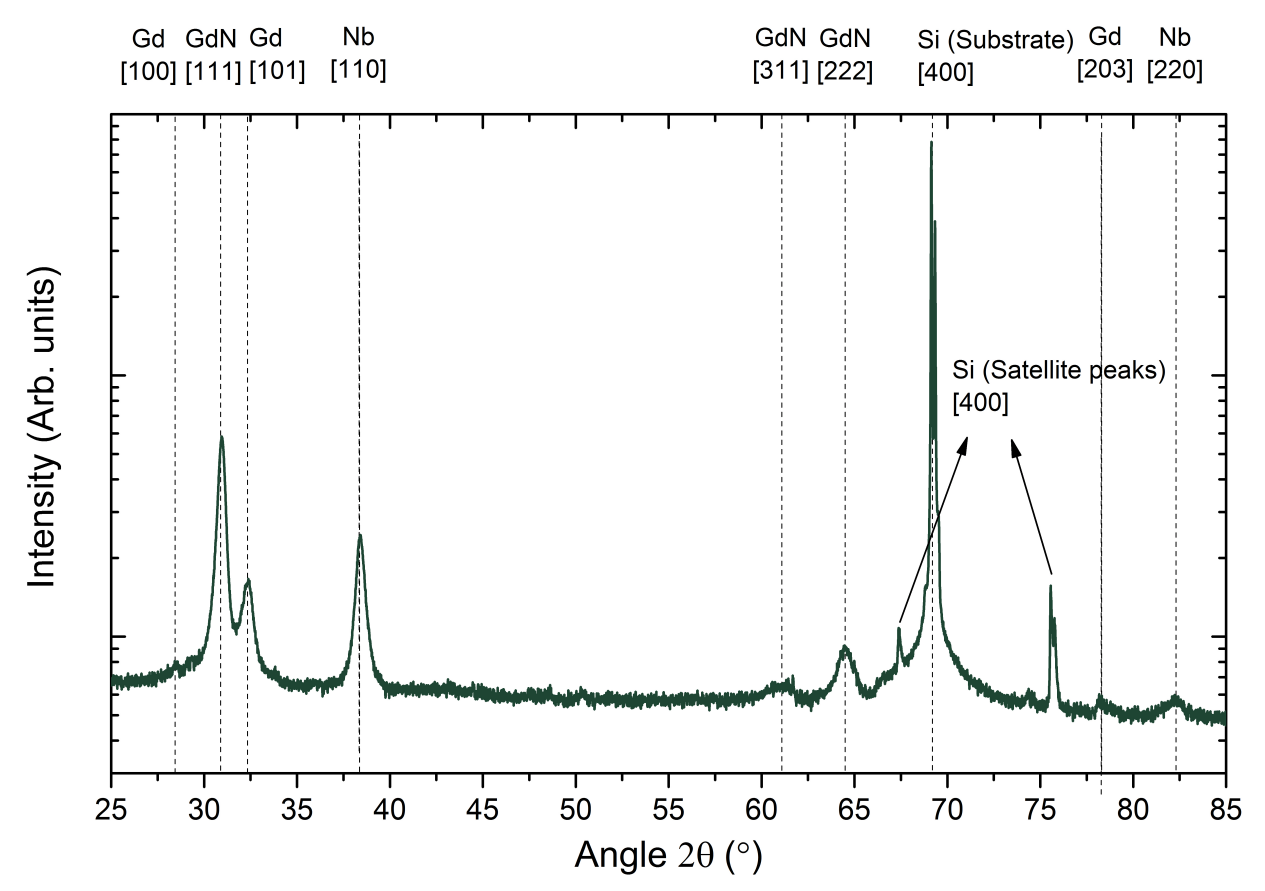

Figure 2.2: XRD $2 \theta$ scan of an MTJ with $\mathrm{Nb}$ bottom contact and Gd top contact (The total structure is $\mathrm{Nb} / \mathrm{GdN} / \mathrm{GaN} / \mathrm{GdN} / \mathrm{Gd}$ all deposited on $\mathrm{SiO}_{2}$ the layer thicknesses of the $\mathrm{GdN}, \mathrm{Nb}$ and $\mathrm{Gd}$ are all $\sim 100 \mathrm{~nm}$. The tunnel barrier is $<5 \mathrm{~nm}$ and does not show a peak on the XRD for this reason.). The peaks are labelled. Small satellite peaks are visible on either side of the $\mathrm{Si}$ substrate peak, these are a result of the incomplete monochromaticity of the $\mathrm{X}$-ray source.

Figure 2.2 shows an XRD spectrum for an MTJ grown on $\mathrm{Nb}$. The peaks are labelled for material and crystal orientation. Successful growth of GdN on $\mathrm{Nb}$ requires the absence of any secondary crystal phases (such as $\mathrm{GdNb}$

\footnotetext{
${ }^{5} h, k$ and $l$ are known as the Miller indices of the crystal planes.
} 
alloys) that form as a result of chemical interaction of the $\mathrm{Nb}$ and GdN at the interface. The peaks shown in the XRD spectrum can all be attributed to the expected growth materials confirming the absence of any secondary alloys or other crystalline phases. The lattice parameter of $\mathrm{Nb}$ provided by Hypres was $a=3.30 \AA$ and the film was strongly textured in the [110] direction normal to the film plane. XRD scans made on films deposited in-house established a lattice parameter of $a=3.29 \AA$ with a standard deviation of $0.02 \AA$. These films were also strongly [110] textured. The quality of the films being similar to those provided by Hypres is encouraging for the integration of $\mathrm{GdN}$ with device-grade $\mathrm{Nb}$.

The peak locations can also be used in conjunction with the peak width to provide information about the size of the crystallites that make up the film. The inference is made using what is known as the Scherrer equation

$$
\tau=\frac{K \lambda}{\beta \cos \theta}
$$

where $\tau$ is the crystallite size, $\lambda$ is the wavelength of the radiation $(1.54 \AA$ for $\mathrm{Cu} K_{\alpha}$ radiation), $\beta$ is the full-width half maximum (FWHM) of the peak in radians and $\mathrm{K}$ is a form factor dependent on the shape of the crystallite (Here taken as 0.9 , as grains are assumed to be spherical). All of the polycrystalline films grown as part of this study had crystallite sizes between 10 and $40 \mathrm{~nm}$ (typically $\sim 20 \mathrm{~nm}$ ).

\section{X-Ray Reflectivity}

The same PANalytical "X'pert Pro" x-ray diffraction setup was also used to perform X-Ray Reflectivity (XRR) measurements. The $\mathrm{Cu}$ target was here used to provide $K_{\alpha}$ radiation at grazing incidence to the plane of the film. Layer thickness and surface roughness of the films can be inferred from the interference fringes of the specularly reflected X-rays. Such a technique is preferable to profilometry as multilayers are able to be assessed. 


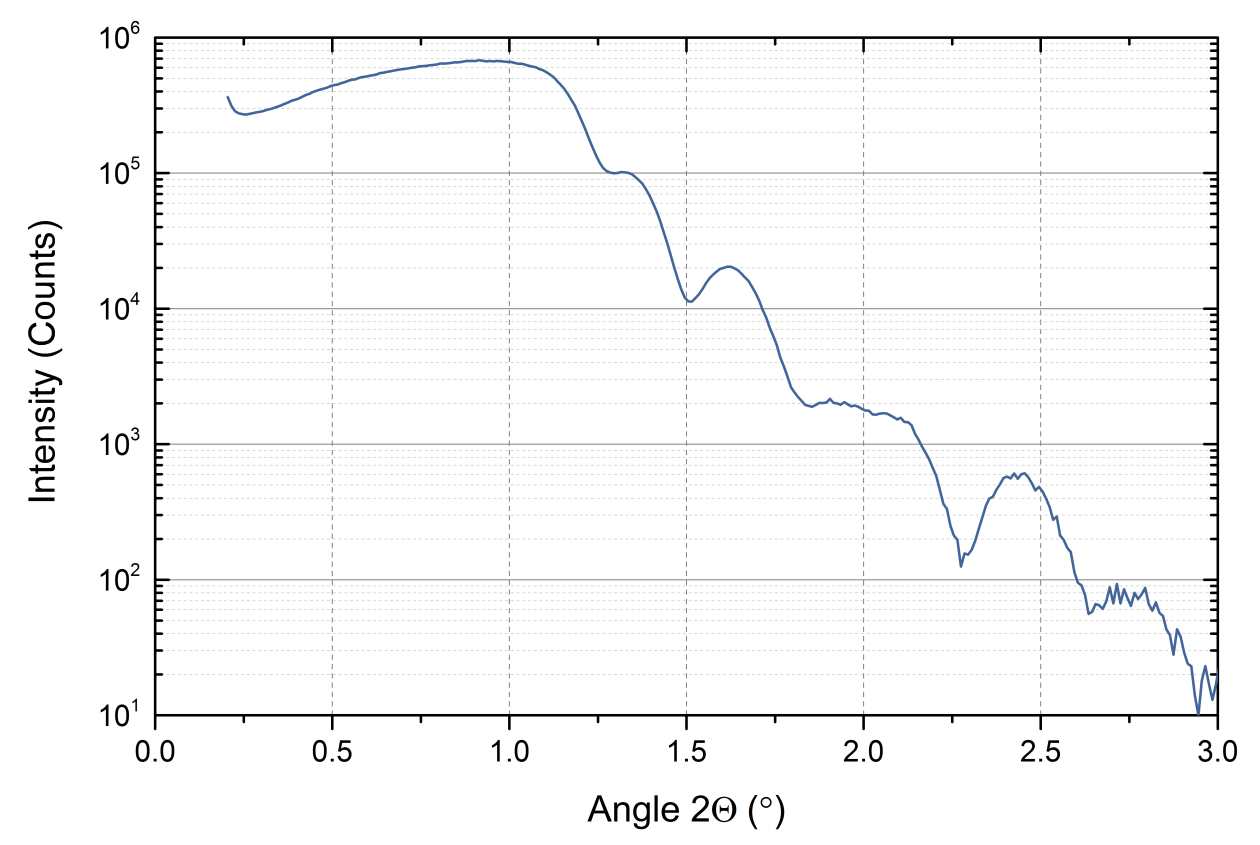

Figure 2.3: XRR scan of a reference sample grown in a typical chrome/gold bottomcontact deposition.

Figure 2.3 shows interference fringes measured using XRR for a chromium/gold deposition on $\mathrm{SiO}_{2}$, such depositions are used as bottom contacts to $\mathrm{GdN}$ for electrical measurements. These interference fringes give information about the layer thickness and the surface roughness of the bottom contact before the sample is returned to the UHV chamber for the growth of the GdN/MTJ. For the sample in Figure 2.3, The chromium thickness was 6.2 $\mathrm{nm}$ and the gold thickness was $25.7 \mathrm{~nm}$, determined by a fit to the data by the XRR analysis software X'pert reflectivity. The RMS surface roughness was $\sim 1.2 \mathrm{~nm}$, determined by the same software.

This study also utilised Scanning Electron Microscopy (SEM) to assess layer thicknesses. However, SEM requires the cleaving of samples to obtain an edge, which is not required for XRR measurements. The propensity of the rare-earth nitrides to oxidise in air along the cleaved edge of 
the sample makes the accuracy of thickness measurements using SEM less certain. However, the decreased efficacy of XRR for films $>80-100 \mathrm{~nm}$ and many-layered films led to SEM being used more commonly to assay the thickness of layered structures.

\section{Atomic Force Microscopy}

In order to grow interfaces that have good contact between the separate thin film layers the growth surface must be very flat. Atomic Force Microscopy was performed using a Nanosurf NaioAFM to assess the growth

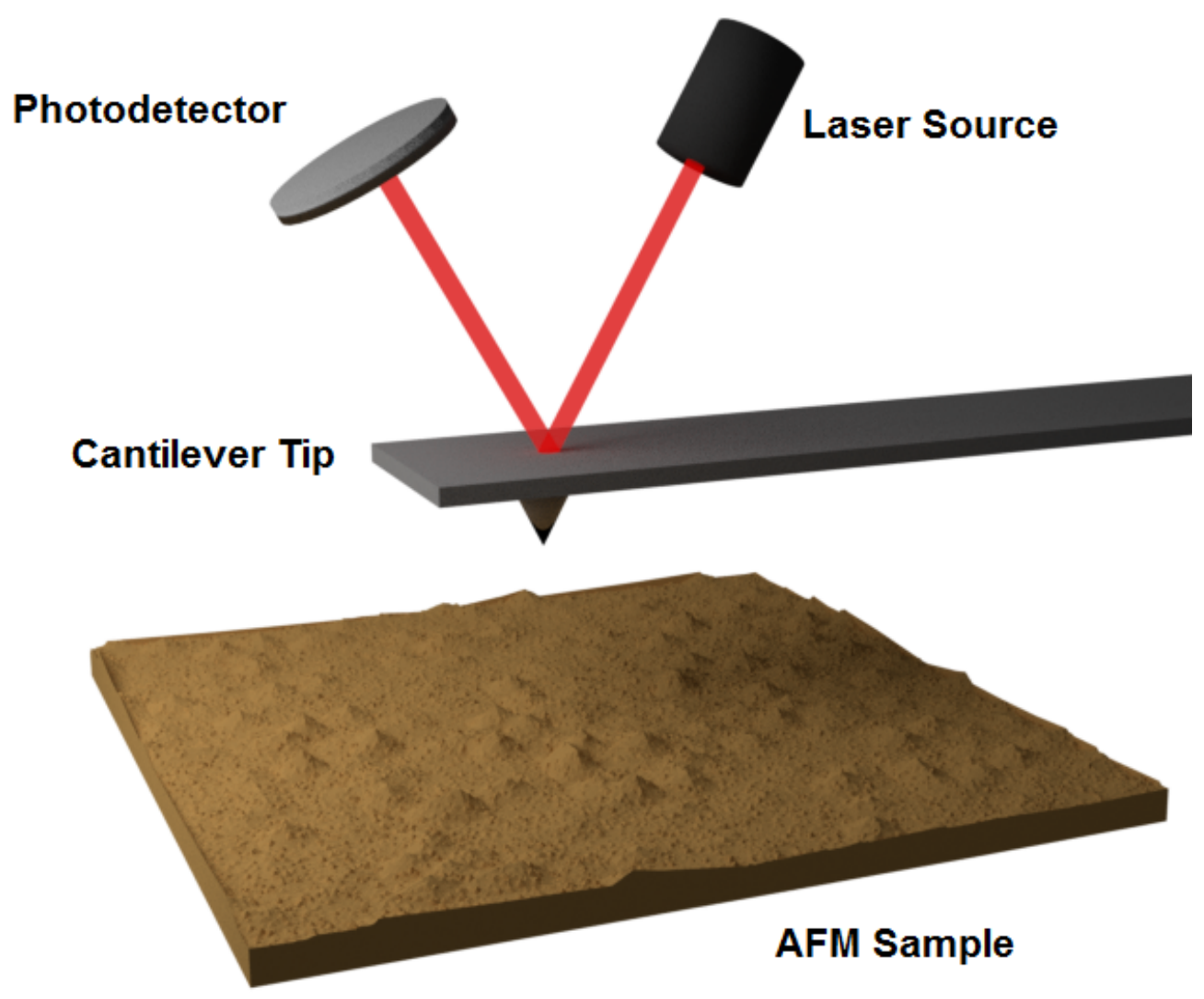

Figure 2.4: Image of the AFM setup showing the "optical lever" that allows very sensitive measurement of the sample height. 
surface quality of bottom contacts prior to GdN/MTJ deposition. This technique involves the scanning of a probe (tip) along the sample maintaining a set force between the probe and surface. Any displacement of the tip is detected by a laser focussed on the back of the cantilever. The reflected beam then falls on a photodetector set away from the sample in a design known as an 'optical lever'. This setup (shown in Figure 2.4) allows detection of incredibly small feature heights.

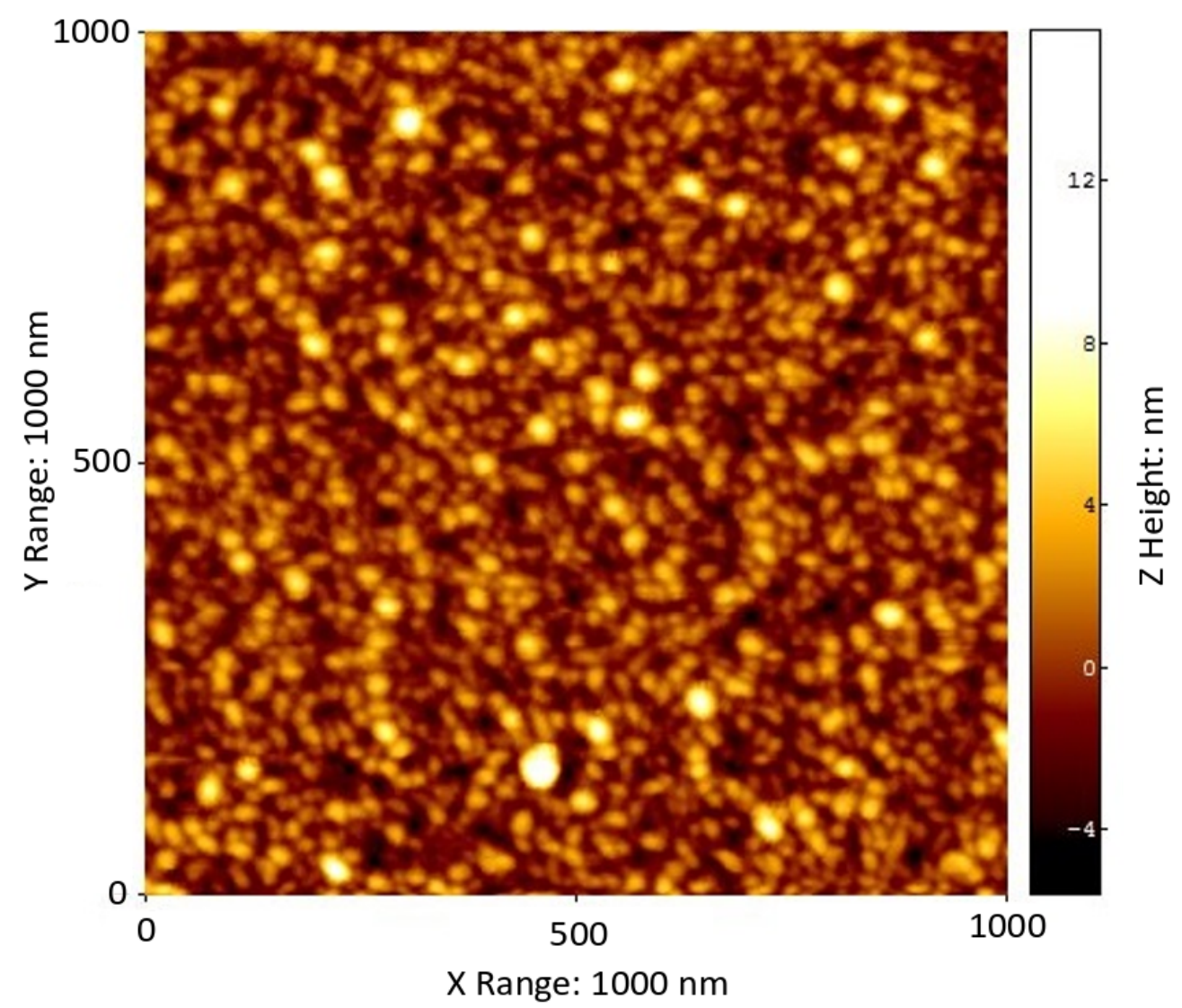

Figure 2.5: AFM surface profile showing the roughness of a $\mathrm{Nb}$ thin film over an area of $1 \times 1 \mu \mathrm{m}$.

The root-mean-square (RMS) surface roughness of the contacts was extracted from the height profile using AFM image processing software 
(SPIP). Large surface roughness of the bottom contact may preclude tunnelling behaviour by causing pinholes in the barrier layer. Pinholes connecting the two electrodes may open conduction channels that subvert the tunnelling process. Figure 2.5 shows a $1 \times 1 \mu \mathrm{m}$ surface scan of a $\mathrm{Nb}$ contact. The surface roughness derived from the scan is $1.84 \mathrm{~nm}$, a value representative of the bottom contact depositions (typically $\sim 2 \mathrm{~nm}$ ). This low surface roughness reduces the probability of pinhole formation, crucial for the growth of reproducible tunnel junctions.

\section{Scanning Electron Microscopy}

Because the wavelength of a high energy electron is much smaller than that of optical light wavelengths, imaging that utilises the interaction of electrons with matter allows the acquisition of images with much higher resolution than those given by typical microscopy. For this reason, an FEI Nova NanoSEM 450 was used to measure the cross-sectional thickness of thin film layers in this project. The structures were prepared for SEM by cleaving using a diamond scribe and were affixed to an aluminium stub using a carbon adhesive tape. The cleaving process exposes the crosssection of the thin film to air before mounting in the SEM vacuum chamber. Samples prepared in this manner are desiccated prior to their introduction to the chamber in order to outgas the samples and tape.

Figure 2.6 shows a typical SEM micrograph with the cross-section of a sample clearly displayed. In this image (An MTJ grown on $\mathrm{Si} / \mathrm{SiO}_{2}$ ) one is able to distinguish the layers clearly as a result of backscatter detection of the probe electrons. Backscatter detection was used as the intensity registered by this technique is proportional to the atomic mass of the target material (Thus heavy metals like $\mathrm{Nb}$ are very bright, $\mathrm{GaN}$ is grey and $\mathrm{SiO}_{2}$ is barely registered). While the backscatter method has poorer resolution than the secondary electron image, the contrast of the different layers allows the determination of their thicknesses to reasonably high accuracy. 


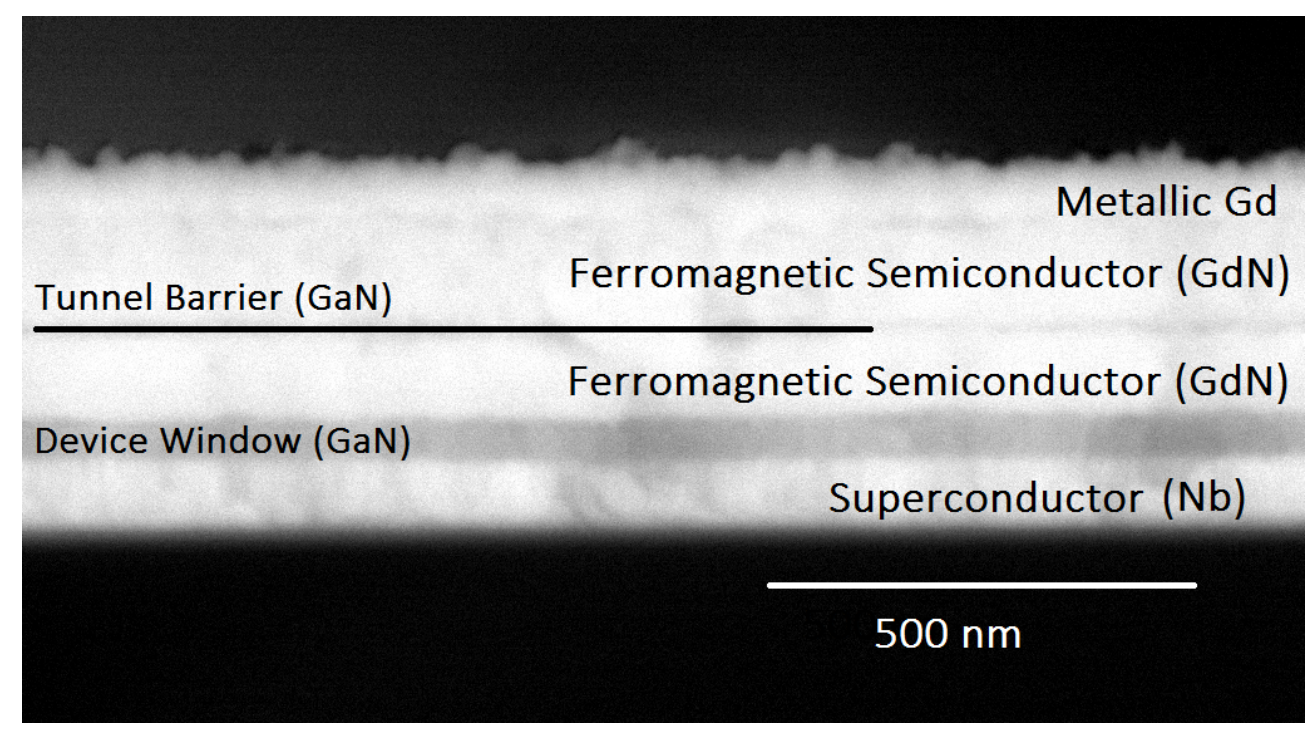

Figure 2.6: SEM micrograph showing an MTJ grown on a thermally oxidised silicon wafer.

\subsection{Developments in Fabrication}

Techniques described in the previous sections (particularly describing measurements using XRD and SEM) confirmed the physical compatibility of $\mathrm{GdN}$ with our metal contact materials ( $\mathrm{Al}, \mathrm{Au}, \mathrm{Gd}$ and $\mathrm{Nb}$ ). Figures 2.2 and 2.6 specifically demonstrate the lack of secondary phases or delamination occurring as a result of chemical reaction between $\mathrm{GdN}$ and $\mathrm{Nb}$. In the initial part of this thesis, electrical measurements were performed on unpatterned films and this lead to issues in the interpretation of the results (as discussed below, see Figure 2.7).

Modelling developed independently of, but equivalent to, reference [42], suggested that current paths in the samples were not well defined and that the voltage decreases as one moves away from the contact injecting current into the metallic films. The relationship between the voltage and the distance is governed by a second order differential equation

$$
\frac{d^{2} V(r)}{d r^{2}}+\frac{1}{r} \frac{d V(r)}{d r}-\frac{1}{t_{G d N} t_{M e t a l}} \frac{\rho_{\text {Metal }}}{\rho_{G d N}} V(r)
$$




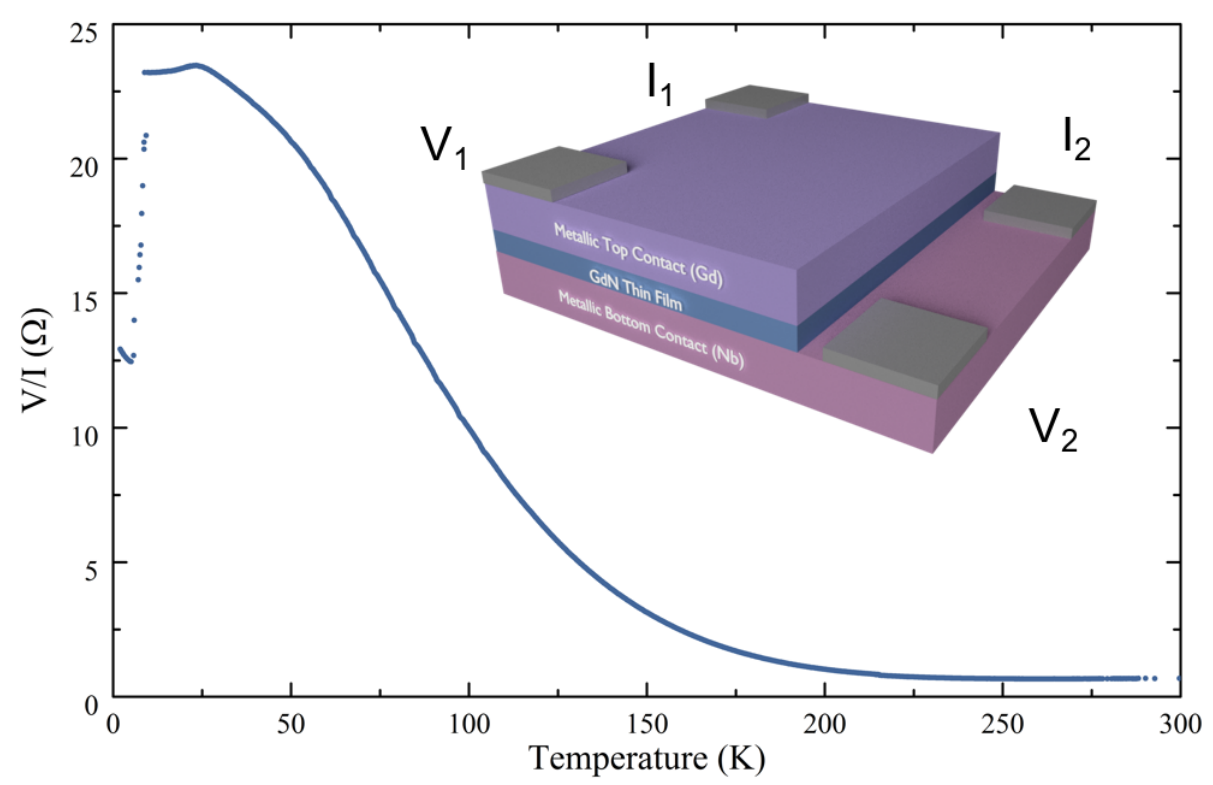

Figure 2.7: Temperature-dependent resistance of a $\mathrm{Nb} / \mathrm{GdN} / \mathrm{Gd}$ structure measured with silver paint contacts applied to the corners of the sample. The inset shows the sample contact geometry, with current running from $\mathrm{I}_{1}$ to $\mathrm{I}_{2}$ and voltage measured between points labelled $V_{1}$ and $V_{2}$.

if one considers the point of current injection to have a circular symmetry (a feature that simplifies the model but gives a useful qualitative result) ${ }^{6}$. The solution to this equation is a modified Bessel function of the second kind with a characteristic length scale given by

$$
\frac{1}{\sqrt{t_{\text {GdN }} t_{\text {Metal }}}}\left(\frac{\rho_{\text {GdN }}}{\rho_{\text {Metal }}}\right)^{\frac{1}{2}} .
$$

Because the length scale is governed by the ratio of the material resistivities, the measured voltage becomes artificially reduced at high tempera-

\footnotetext{
${ }^{6}$ In equation (2.4) $V(r)$ is the voltage at a radial distance of $r$ from the point of current injection, $t_{G d N}$ and $t_{M e t a l}$ are the thicknesses of the $\mathrm{GdN}$ and metal layers respectively and $\rho_{G d N}$ and $\rho_{\text {Metal }}$ their resistivities.
} 
ture as $\rho_{G d N}$ and $\rho_{\text {Metal }}$ become comparable. This is visible in Figure 2.7, where the resistance appears to go to zero near room temperature. The failure of the metallic contacts to behave as equipotentials is a geometric issue with the device structure and developments were made to the fabrication process in order to more accurately perform electrical measurements.

To help define the geometry, an evaporated gold contact was deposited atop the capping layer in an ex-situ process. Making contact to the gold, with voltage and current leads close together (see inset to Figure 2.8), improved the accuracy of the voltage measurement, with the temperaturedependent resistance (see Figure 2.8) not significantly effected by the aforementioned geometric effects.

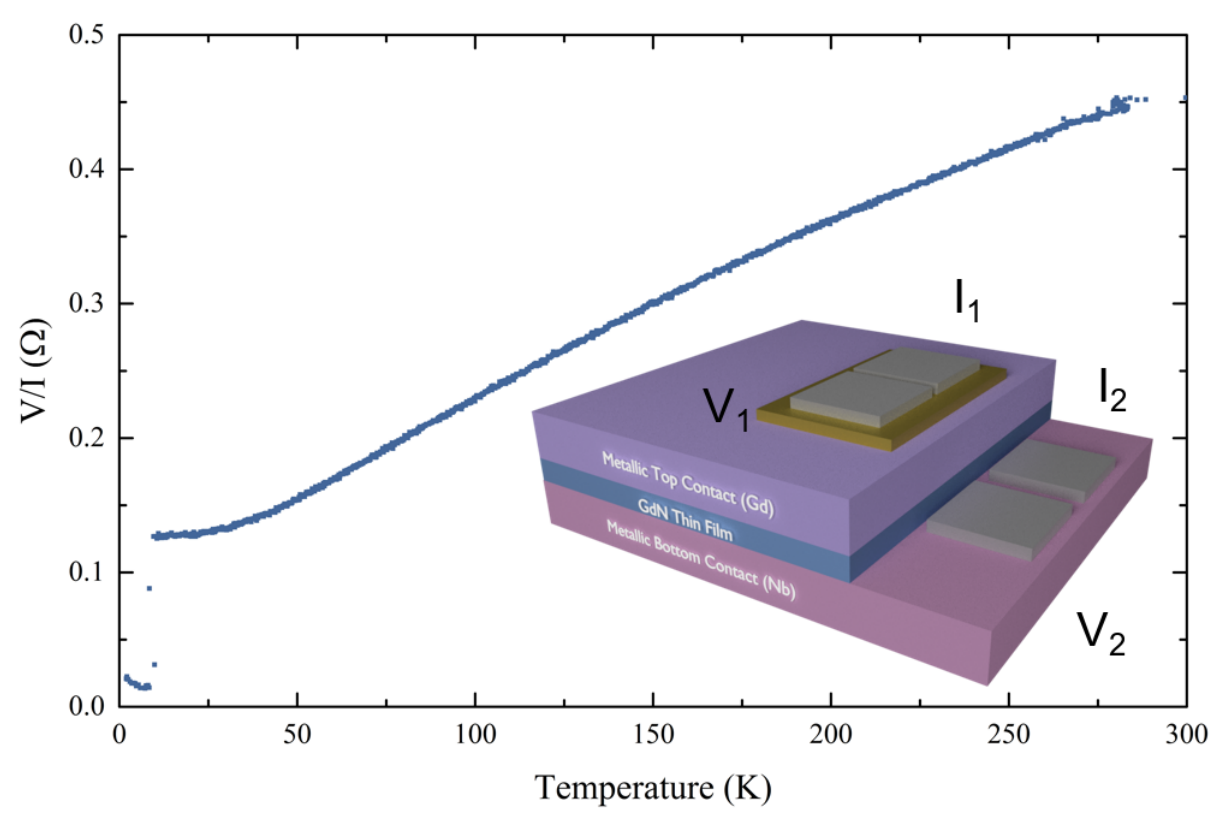

Figure 2.8: Temperature-dependent resistance of a $\mathrm{Nb} / \mathrm{GdN} / \mathrm{Gd}$ junction with a gold top contact applied. The inset shows the sample contact geometry, with current running from $I_{1}$ to $I_{2}$ and voltage measured between points labelled $V_{1}$ and $V_{2}$. 
The temperature-dependent resistance shown in Figure 2.8 was strongly metallic, suggesting the resistance was dominated by transport through a metallic part of the structure. The discontinuity at the superconducting transition temperature of $\mathrm{Nb}$ confirms that the signatures of CPP electrical transport through the GdN layer were obscured by lateral movement of the current in the plane of the $\mathrm{Nb}$. This imposed a requirement for more robust engineering of structures allowing measurement of electrical characteristics perpendicular to the plane of the film.

The early electrical measurements described above catalysed the development of a lithographic fabrication process, due to the problems with current spreading in metallic layers and the strong in-plane contribution to measurements. The following section describes this lithography process and photomask I designed to circumvent these issues.

\subsubsection{Optical Lithography}

Photolithography was used to define the geometry of all subsequently grown samples. In this process a thin layer of UV-active polymer (photoresist AZ1518) is coated onto the substrate. The exposure of the photoresist to UV-light effects a chemical change which allows the exposed section to be removed by an alkali developer solution (AZ351B). Optical lithography was used to develop cross-contact device geometries with lateral dimensions ranging from 60 microns down to 10 . This geometry allows accurate four terminal electrical measurements to be made through the device structure (that is, with the current perpendicular to the plane of the film) by growing bottom and top contact layers in separate growths and using the photolithography process to ensure a well defined area of overlap where the current can pass from bottom to top.

Production of device geometries is accomplished by the use of a photomask designed to transfer patterns onto the substrate. Using the lithographic process areas of the substrate can be made to retain the photore- 
sist layer during the growth process, this is removed after the growth of the metal or REN layer by a "liftoff" step (here the dissolving of the remaining photoresist in acetone or heated n-methyl-2-pyrrolidone [NMP]) taking the film deposited on top of the photoresist with it, but leaving the metal/REN layers on the part of the substrate previously developed.

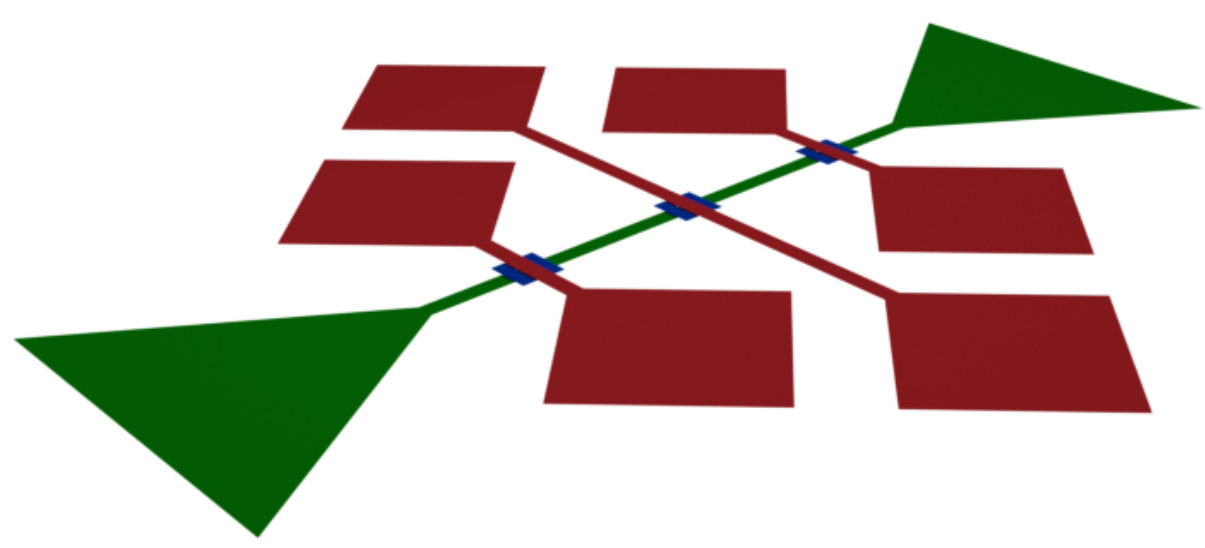

Figure 2.9: Schematic of the new mask designed for this project. The bottom contact is shown in green and the top contact in red. The blue indicates the position of the GaN windows at the crossing of the contacts, defining the active area of each device.

In the interests of increasing throughput, preventing device oxidation and increasing ease of mask alignment; a new mask was designed that allowed the growth of three separate devices on a $1 \mathrm{~cm}^{2}$ substrate (see Figure 2.9 $)^{7}$. Each device of the three was designed to have a different active area in order to push to the fabrication of smaller devices, important for technological application. Active device areas have lateral dimension of

\footnotetext{
${ }^{7}$ The mask developed as part of this project was a direct response to early electrical measurements and struggles to interpret and model them. Previous photolithography attempts gave only one device per $1 \times 1 \mathrm{~cm}$ chip, did not utilise the $\mathrm{GaN}$ window used to protect against oxidation from the sample edges and were $\sim 20$ times bigger than the devices allowed by the new mask. The new mask was manufactured by Andrew Best at Callaghan Innovation using electron beam lithography.
} 
$60,40,30,20$ and 10 microns defined during growth by the deposition of a $40 \mathrm{~nm}$ layer of insulating $\mathrm{GaN}$ in a square shape isolating the top and bottom contacts from one another, but allowing contact within the active area of the device. A top-down view of a GaN window is shown in Figure 2.10 .

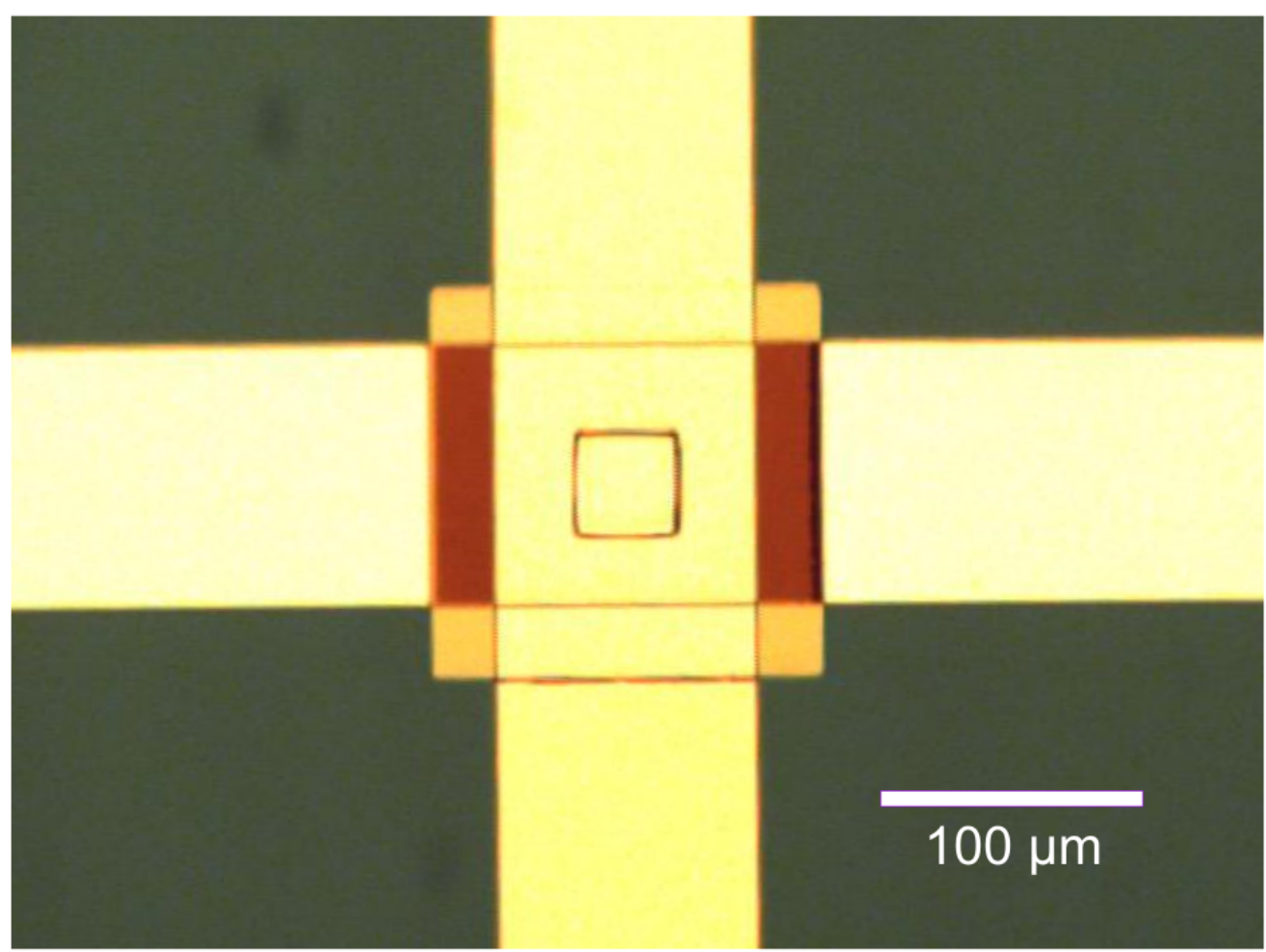

Figure 2.10: A typical device after the growth of all three layers, showing the top-down view of the device shape. The bottom contact runs horizontally across the image, while the top contact runs vertically. The current passes between the top and bottom contacts through the 40 micron device at the centre defined by GaN isolating square. 


\subsection{Interpretation of Electrical Measurements}

The majority of electrical measurements included in this thesis tend to be one of two varieties: Current-Voltage (or IV) curves and temperaturedependent resistance. The IV curves of a sample give an indication of the mode of electrical transport dominating the measurement. This gives insight into the conduction across material interfaces. In samples such as the ones grown for this work, linear relationships between current and voltage indicate ohmic conduction across interfaces, meaning that the sample obeys Ohm's law. Non-linear relationships between current and voltage invoke a need for more sophisticated electrical transport models.

An IV curve is included below showing an in-plane (CIP) measurement of a $90 \mathrm{~nm}$ thick $\mathrm{Nb}$ film grown in the same conditions as the contacts for CPP device structures. That the IV curve is linear indicates ohmic contact between the silver paint contacts and the niobium film.

Because this thesis involves the electrical measurement (in a CPP geometry) of devices of varied sizes, these measurements are generally presented as JV curves, where the voltage is plotted against the current density (the measured current divided by the area of the device, assumed to be the same area as the window of $\mathrm{GaN}$ defined by the lithography). This change ensures that all electrical transport measurements are normalised with respect to device area, meaning that samples grown in the same deposition should exhibit curves that are independent of device area.

The other variety of electrical measurement plotted for multiple samples is the temperature-dependent resistance. These plots show the dependence of the resistance (or, more properly, $\mathrm{V} / \mathrm{I}$ as resistance is only meaningful in the context of Ohm's law, or samples with linear IV curves) on the temperature, illuminating whether the sample is, for example, semiconducting or metallic. It is also possible to recognise phase changes such as the transition to superconductivity (where the resistance falls to zero below a critical temperature, $T_{C}$ ). Figure 2.12 shows an example of this for 


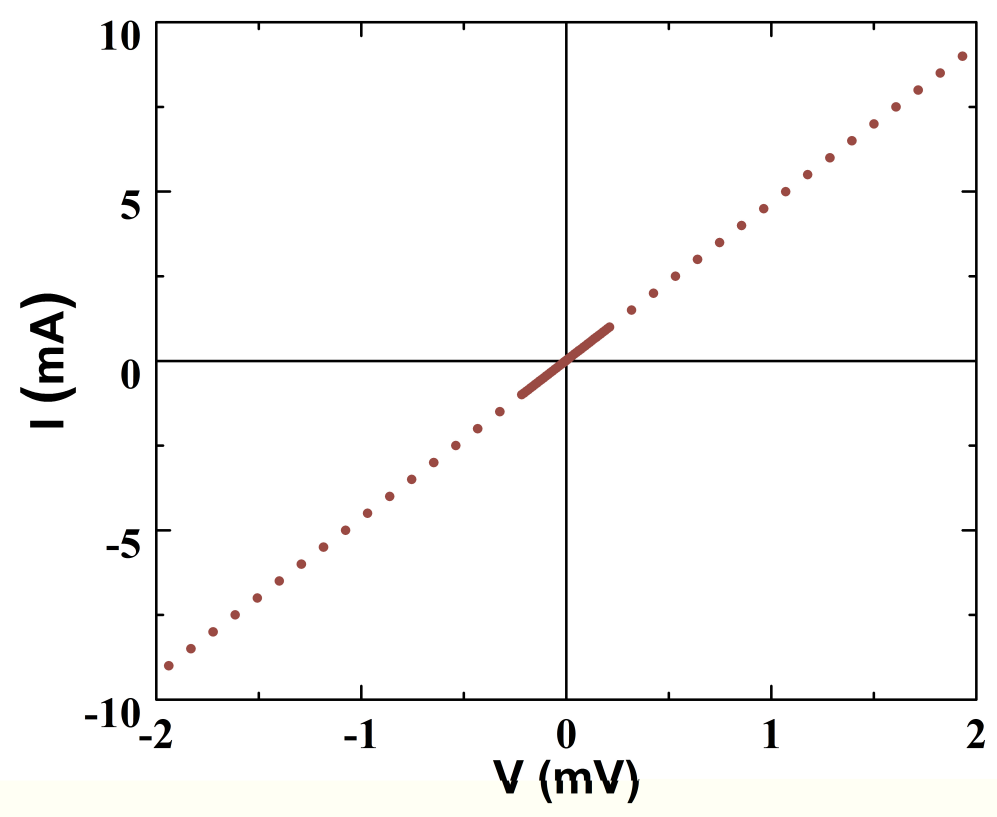

Figure 2.11: A room temperature IV measurement of a $90 \mathrm{~nm}$ thick $\mathrm{Nb}$ film in a CIP geometry. The relationship is clearly linear, indicating ohmic contact.

niobium grown under the same conditions as the CPP device structures. The superconducting transition is clearly visible at $6 \mathrm{~K}$.

For the same reason as for the IV curves, it is necessary to adapt the temperature-dependent resistance in order to remove the effect of device size on the result. To do this, temperature-dependent resistance measurements have been plotted as $\mathrm{V} / \mathrm{J}$ rather than the actual sample resistance. For samples with linear IV curves, $\mathrm{V} / \mathrm{J}$ is equivalent to what is known as the resistance-area product.

$$
\frac{V}{J}=\frac{V}{I} \times A=R A
$$

As part of this thesis, two different physical effects have been introduced that control the relationship between the voltage applied to the 


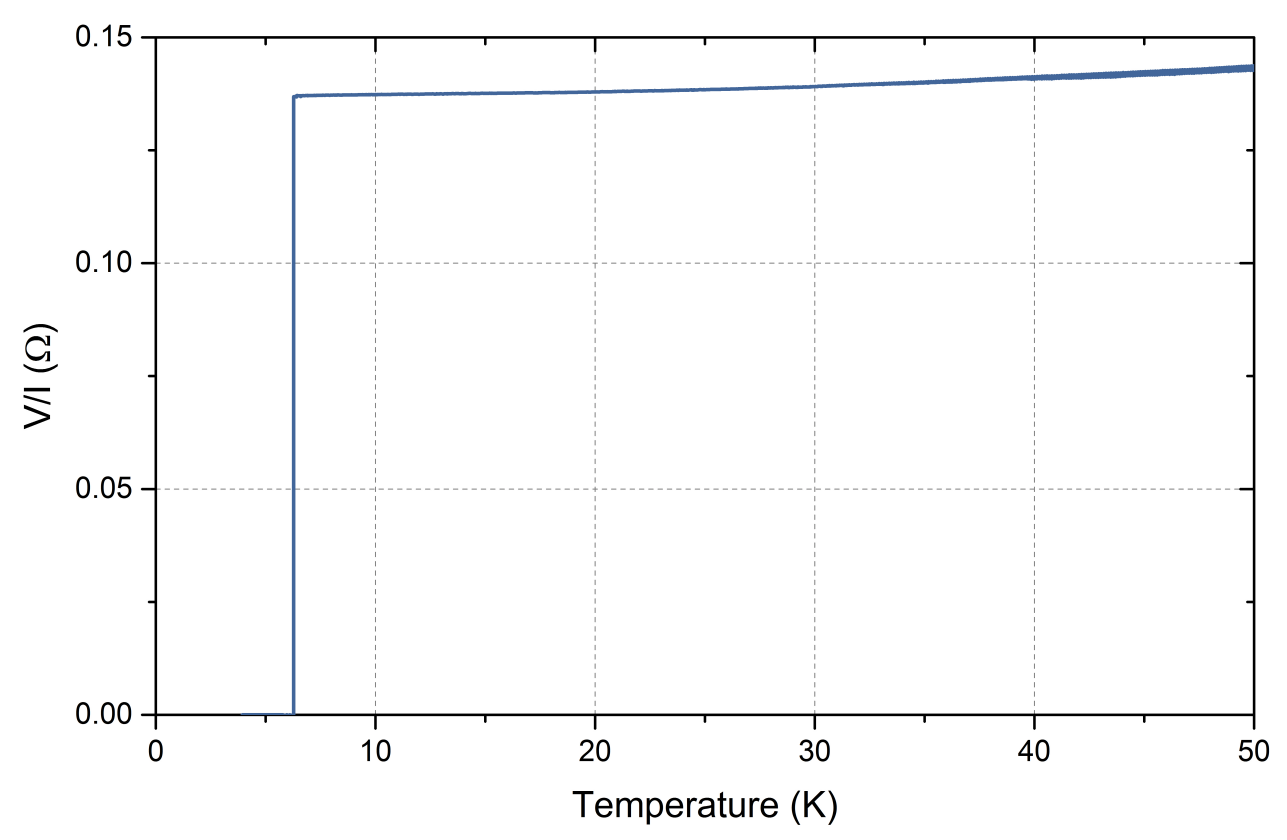

Figure 2.12: Temperature-dependent resistivity measurement of $\mathrm{Nb}$ showing the superconducting transition at $6 \mathrm{~K}$.

sample and the measured current density (given the linearity of transport through bulk GdN). Firstly, there is the clear non-linearity of insulating barriers such as the GaN introduced in MTJ devices. The other is the behaviour of the material interfaces of the structure. The impact of these interfaces on the JV characteristics of a device depends strongly on whether these interfaces exhibit ohmic (linear) or non-ohmic (non-linear) conduction behaviour.

Metal/semiconductor interfaces are notorious for their propensity to form barriers to electron conduction. Such barriers were named Schottky barriers following the first successful theoretical description of the effect and found heavy application in electrical devices such as diodes before the advent of p-n junctions. The dominance of the MTJ tunnelling barrier over the device electrical characteristics is shown beyond a doubt by 
the comparison of the resistance-area product of device structures with and without $\mathrm{GaN}$ introduced. Given the intended device functionality, the inconsequence of series resistances that result from transport through the GdN layers and across the metal/GdN interfaces is a positive result. Herein, the discussion turns to the various descriptions that are used to model both insulating barriers and Schottky interfaces, in the hope that such models can provide useful information about the properties of these devices.

\subsubsection{Schottky Contacts}

The Schottky model of metal/semiconductor interfaces is much broader than the facets of it that are introduced here. While effects like charge accumulation lead to interesting effects like the development of the 2 dimensional electron gas, the intrinsic n-type doping of $\mathrm{GdN}$ and the relation of the Fermi level in GdN to that of the metals involved here renders the depletion of the conduction band the most relevant aspect of the theory ${ }^{8}$. For a more comprehensive discussion see the book "Semiconductor Material and Device Characterisation" by Schroeder or "Physics of Semiconductor Devices" by Sze[43, 44].

Where a metal/semiconductor interface is formed, in the case that the work function of the metal is greater than that of the semiconductor, it is possible for the semiconductor electrons close to the interface to find lower energy states within the metal and move to occupy them. This behaviour equalises the Fermi levels of the two materials as the process approaches an equilibrium, however the excess negative charge now residing in the metal now provides a significant potential barrier for electrons passing across the interface(see Figure 2.13). The barrier height, $\varphi_{B}$, is given by the work function of the metal, $\varphi_{M}$, less the electron affinity of the semicon-

\footnotetext{
${ }^{8}$ It may be, for example, that the $\mathrm{Al} / \mathrm{GdN}$ behaves as an accumulation layer. However, this does not effect the conduction perpendicular to the plane of the film and so behaves effectively as an ohmic contact in this context.
} 


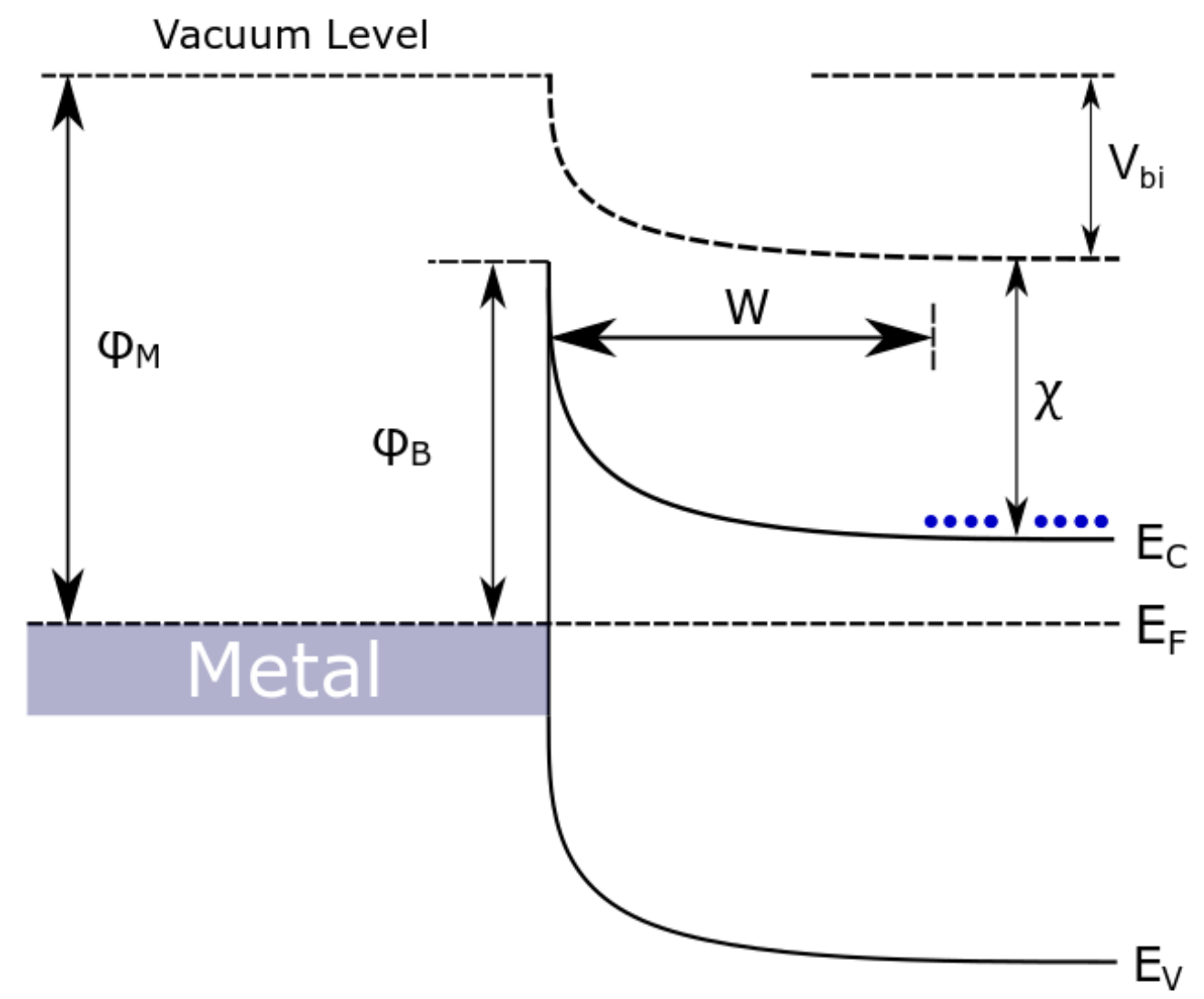

Figure 2.13: Image (not to scale) showing the depletion mode of a simple Schottky contact. $E_{C}$ and $E_{V}$ are the levels of the conduction and valence band respectively. $E_{V}$ is the Fermi level. The electron affinity is indicated by $\chi$. W gives the depletion length away from the interface. The work function of the metal and the height of the Schottky barrier are given by $\varphi_{M}$ and $\varphi_{B}$ respectively. Finally $\mathrm{V}_{\mathrm{bi}}$ is the built in potential difference, reflecting the difference in the energy between the materials' bulk Fermi energies

ductor, $\chi$ (the potential difference between the semiconductor conduction band and the vacuum level).

$$
\varphi_{B}=\varphi_{M}-\chi
$$

Because electrons leave a region of the bulk semiconductor, there is a remnant "depletion zone" (indicated in Figure 2.13 by a W) lacking charge 
carriers. Such a zone can act as an impediment to conduction in cases that the length of this depletion area is long or the barrier high.

Passing of current through such a contact relies on one of two methods: thermionic emission, where the carrier has adequate thermal energy to overcome the barrier; or field emission where the carrier tunnels quantum mechanically through the barrier. The latter case leads to strongly non-linear JV characteristics while the former can approximate ohmic behaviour where the barrier height is low and the temperature of the sample provides sufficient energy to carriers (the thermionic emission current $J \propto \exp -q \varphi_{B} / k_{B} T$ approaches unity in this limit). Generally conduction happens as a result of both these methods.

\subsubsection{The Simmons Model}

General tunnelling of electrons through potential barriers has been studied since the 1930s[45, 46]. One of the more popular models to describe tunnelling in practical systems is that developed by Simmons in the 1960s $[11,12]$. This model relates measurable quantities, the voltage across the tunnel barrier and the current density passing through it, to parameters that describe the tunnel barrier (its width in real space and the 'height' of the potential barrier). The simplicity of this model and its relation of the barrier features to practically measurable quantities make it an ideal tool to begin to interpret the electrical behaviour of the devices.

A schematic showing the bands of the electrodes and tunnel barrier is shown in Figure 2.14. In the absence of an applied voltage the barrier approximates a rectangular shape (complicated by band bending effects at the interfaces). The Fermi level aligns across the device structure and the conduction band edges of the GdN are matched either side of the barrier. Applying a voltage across the device deforms the energy bands of the barrier and electrode (in a way that is accounted for when applying the Simmons model). This induces electron tunnelling through the GaN bar- 


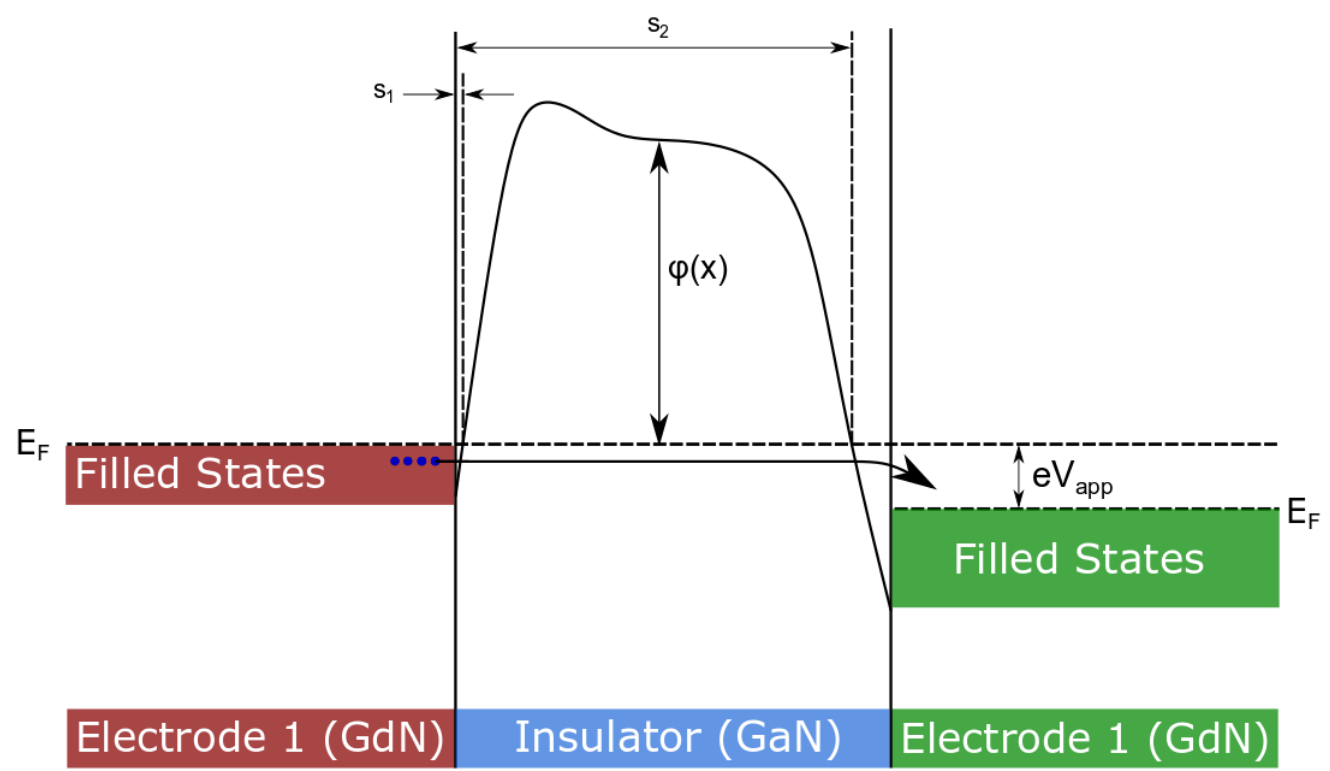

Figure 2.14: Schematic of the material band structures at the tunnel barrier of an MTJ. This image shows the parameters used in the Simmons model. $\Delta s=s_{2}-s_{1}$ is the width of the tunnel barrier at the Fermi level. $\varphi(x)$ is the height of the potential barrier and $\mathrm{V}_{a p p}$ is the applied voltage.

rier from one GdN electrode to the other. The Simmons model is able to account for the different voltage regimes, symmetric/antisymmetric barriers and the electrodynamic effects (image forces) that govern the tunnelling behaviour.

In the case of an approximately rectangular barrier (the relative size of the band gaps of GdN and GaN coupled with the device size ensure this here) at zero temperature, the Simmons model gives a current density of

$$
\begin{array}{r}
J\left(V_{a p p}, T=0\right)=\frac{6.2 \times 10^{10}}{s^{2}}\left[\left(\varphi_{0}-V_{a p p} / 2\right) \exp -1.025\left(\varphi_{0}-V_{a p p} / 2\right)^{\frac{1}{2}}-\right. \\
\left.\left(\varphi_{0}+V_{\text {app }} / 2\right) \exp -1.025\left(\varphi_{0}+V_{a p p} / 2\right)^{\frac{1}{2}}\right]
\end{array}
$$


where $\mathrm{s}$ is the physical width of the tunnel barrier, $\mathrm{V}_{a p p}$ is the applied voltage and $\varphi_{0}$ is the height of the potential barrier ${ }^{9}$. The numerical constants above provide for $\mathrm{s}$ to be measured in Angstroms and $\varphi_{0}$ measured in electron volts.

Accounting for the image charges the electron 'sees' in the electrodes (image charges are imaginary charges that are used to imitate the effect of certain boundary conditions in electrodynamics problems) when tunnelling through the barrier provides a minor correction to the prediction of the Simmons model. Generally, the inclusion of the image charges tends to 'round off' the edges of the potential barrier.

This effect is included in the model by adjusting the barrier height to $\varphi_{I}$.

$$
\varphi_{I}=\varphi_{0}-\left(\frac{V_{a p p}}{2 s}\right)\left(s_{1}+s_{2}\right)-\frac{5.75}{K\left(s_{2}-s_{1}\right)} \ln \left[\frac{s_{2}\left(s-s_{1}\right)}{s_{1}\left(s-s_{2}\right)}\right]
$$

Here $\mathrm{K}$ is the dielectric constant of the barrier material ( 12 for $\mathrm{GaN}$ ) and $s_{1}$ and $s_{2}$ are the endpoints of the potential barrier at the Fermi level. Treating the barrier as an approximately rectangular barrier gives values of

$$
s_{1}=\frac{6}{K \varphi_{0}}
$$

and

$$
s_{2}=s\left[1-\frac{46}{3 s K \varphi_{0}+20-2 s K V_{a p p}}\right]+\frac{6}{K \varphi_{0}}
$$

for $s_{1}$ and $s_{2}$.

Making these adjustments to the barrier height alter the model so it now gives

$$
\begin{aligned}
& J\left(V_{a p p}, T=0\right)=\frac{6.2 \times 10^{10}}{\Delta s^{2}}\left[\varphi_{I} \exp -1.025 \Delta s\left(\varphi_{I}\right)^{\frac{1}{2}}-\right. \\
& \left.\left(\varphi_{I}+V_{\text {app }}\right) \exp -1.025 \Delta s\left(\varphi_{I}+V_{\text {app }}\right)^{\frac{1}{2}}\right]
\end{aligned}
$$

\footnotetext{
${ }^{9} \varphi_{0}$ replaces $\varphi(x)$ in the case that the barrier is approximated as near-rectangular. The Simmons model has a wide range of applicability and $\varphi(x)$ refers to the case of an arbitrary barrier.
} 
as the current density associated with an applied voltage, $\mathrm{V}_{a p p}$, at zero temperature.

To understand the characteristics of the devices measured the model must incorporate some temperature dependence. This is done by making a correction to the model

$$
\frac{3 \times 10^{-9} \times \Delta s^{2} T^{2}}{\varphi_{0}-V_{a p p} / 2}
$$

which runs to second order in temperature. The equation used to fit the $\mathrm{J}-\mathrm{V}$ characteristics of the tunnel junctions was then (with correction):

$$
J\left(V_{a p p}, T\right)=J\left(V_{a p p}, 0\right)\left[1+\frac{3 \times 10^{-9} \times \Delta s^{2} T^{2}}{\varphi_{0}-V_{a p p} / 2}\right]
$$




\section{Chapter 3}

\section{GdN structures with Metal Contacts}

The REN's susceptibility to oxidation is well known[21] and GdN is no exception to the rule. To prevent this, a protective layer must be deposited in the growth chamber before removing the sample from vacuum. Within the context of electrical measurement, conduction properties of the capping layer are of particular importance. Characterisations of the electrical properties of RENs have historically been performed using in-plane (CIP) measurements with the Hall-bar or Van der Pauw geometries, for these characterisations insulating capping layers are required and materials such as $\mathrm{GaN}$ and AlN are commonly used. In electrical measurements perpendicular to the plane (a CPP geometry), a rare case in the REN literature, metallic capping layers are required to electrically contact the REN. This chapter explores the use of aluminium and gadolinium metal as metallic contact layers to GdN in a CPP geometry, firstly as a single layer and then as part of a MTJ device. 


\subsection{GdN with Al contacts}

To explore the contact formed between $\mathrm{GdN}$ and $\mathrm{Al}$, vertical structures were grown on $\mathrm{SiO}_{2}$. These consisted of a single 100nm thick layer of GdN between two 100nm Al contacts. The IV curves of the samples were measured by passing current through the structure in a CPP geometry at $4 \mathrm{~K}$. The assumption that the area of each device is the same as the corresponding window size on the lithographic mask was used to derive a current density and this was plotted against the voltage across the device in Figure 3.1. The striking feature of this plot is the failure of the two JV curves to overlay. As discussed in the previous chapter, the application of a voltage to any conducting device is met with the development of a current density (for ohmic materials this manifests as $J=\sigma E$ where sigma indicates the conductivity of the material, a tensor in general, and $\mathrm{E}$ is the applied electric field).

In Figure 3.1, the devices measured have sizes of $20 \times 20 \mu \mathrm{m}^{2}$ (the squares) and $10 \times 10 \mu m^{2}$ (the circles). Because the conductivity is independent of the sample geometry, the failure of the JV curves to overlay suggests that the area used to derive the current density (the nominal area of the device) is not representative of the area through which the current passes.

To address this, we assumed that part of the nominal area, a strip following the perimeter of the device area (such a non-conductive strip may for example occur as a result of the lithography methods, the perimeter of the GdN may become oxidised etc.), was non-conducting. Such an effect would reduce the active area of the device. By assuming the same strip width for each device ( $\sim 3 \mu \mathrm{m}$ so side length is $\mathrm{L}-6 \mu \mathrm{m}$ where $\mathrm{L}$ is the nominal side length) we were able to approximate each device area and find where the dependence of the current density on the applied voltage is identical across devices. This method of approximation of the area allows the identification of a JV curve independent of the area of the device. 


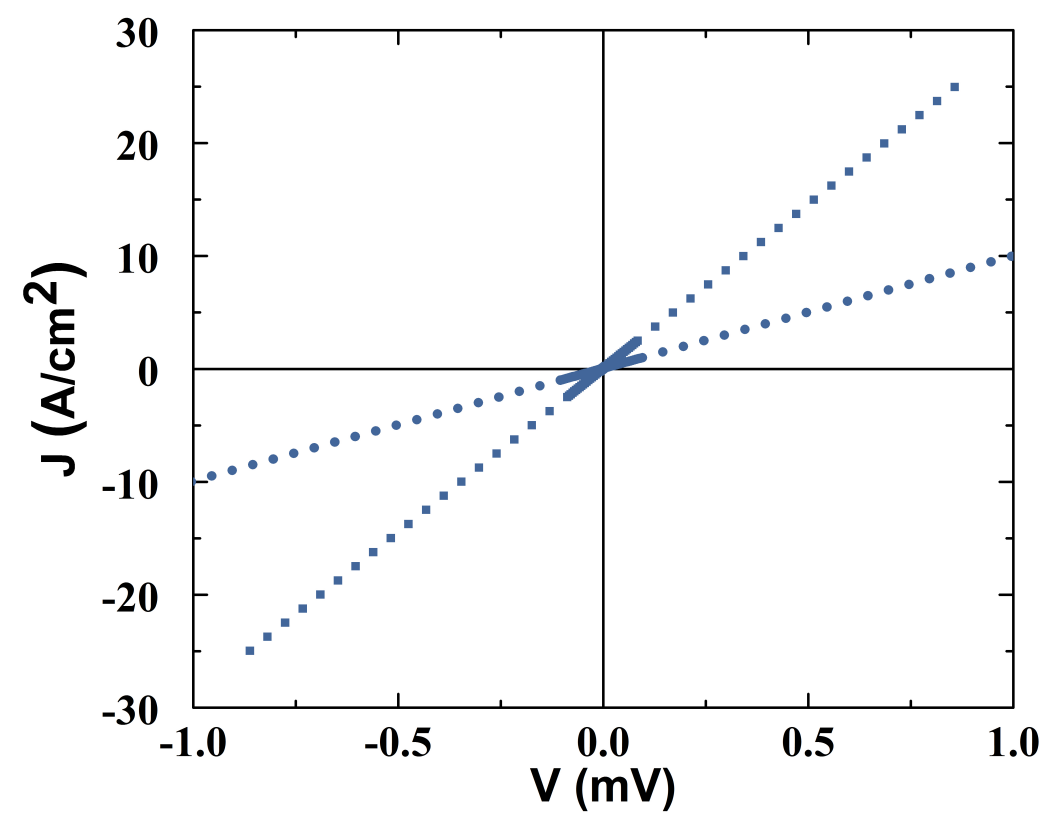

Figure 3.1: JV characteristics of GdN at $4 \mathrm{~K}$, measured in a CPP geometry with $\mathrm{Al}$ contacts. Two samples are shown. The squares represent data taken for a device with area $20 \times 20 \mu \mathrm{m}^{2}$ while the circles show data from a device with area $10 \times 10 \mu \mathrm{m}^{2}$.

Figure 3.2 shows the JV characteristics of the Al-contacted GdN after adjustment has been made to correct the device area. The figure shows a clear linearity, indicating an ohmic contact at the interface between the two materials. The ohmic nature of the contact establishes $\mathrm{Al}$ as an excellent metal for contacting GdN and allows the determination of some material properties of the GdN layer.

Making a least-squares linear fit to the IV plot returns a resistance of $10.2 \Omega$ for the structure. Assuming that the resistance is dominated by transport through the GdN layer rather than the metallic contacts allows an estimate of the resistivity of the GdN layer as the geometry of the structure (the thickness and area of the GdN layer) is known. 


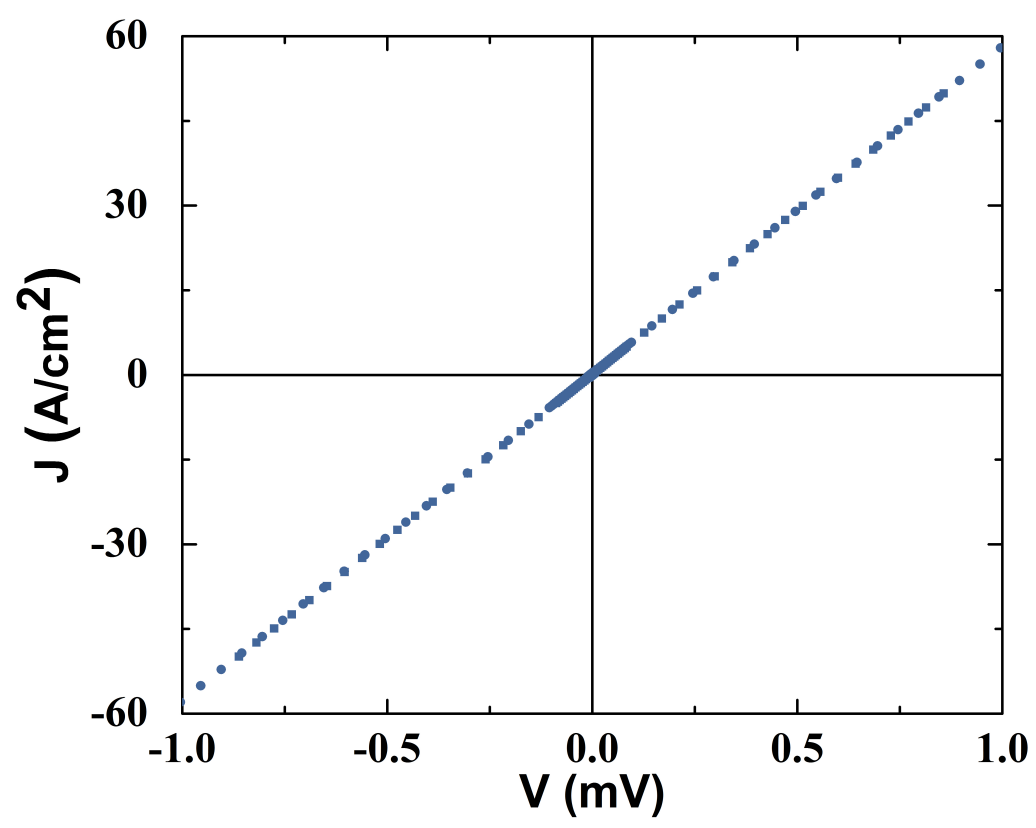

Figure 3.2: JV characteristics of GdN in a CPP geometry with $\mathrm{Al}$ contacts. The squares and circles represent the same devices as in Figure 3.1 with the area adjusted to account for the non-conducting strip.

$$
\rho=\frac{R A}{t}
$$

Using Equation 3.1 puts the resistivity of the GdN layer at $\sim 3 \Omega . \mathrm{cm}$ at $4 \mathrm{~K}$. Here it is instructive to compare this value with the abundant in-plane GdN resistivity values quoted in the literature[47, 48]. Films produced in growth conditions similar to this study have had measured resistivities anywhere between $\sim 1 \mathrm{~m} \Omega . \mathrm{cm}$ and $5 \Omega$.cm. These numbers provide no evidence that the resistivity of polycrystalline GdN differs between in-plane and perpendicular-to-plane measurements.

Temperature-dependent resistance measurements of the $\mathrm{Al} / \mathrm{GdN} / \mathrm{Al}$ structure were also carried out (not shown) and contain a peak in the data around the Curie temperature of GdN. This feature is also visible in elec- 
trical characterisations of GdN measured in-plane (CIP), supporting the assertion the electrical behaviour of $\mathrm{GdN}$ is similar for both geometries.

Calculating V/J (the resistance-area product for this structure, see equation 3.2) from the temperature-dependent resistance measurement gives a value of $32 \mu \Omega . \mathrm{cm}^{2}$. This derived quantity (motivated at the end of the previous chapter) provides a useful means of comparison across devices and will be referenced in later sections.

$$
\frac{V}{J}=\frac{V}{I} \times A=R A(\text { For ohmic conductors } \mathrm{V} / \mathrm{I}=\mathrm{R})
$$

Another material for which contact behaviour is of particular interest is gold $(\mathrm{Au})$, which forms a good ohmic contact to many semiconducting materials and has been shown to form an ohmic contact to GdN in planar

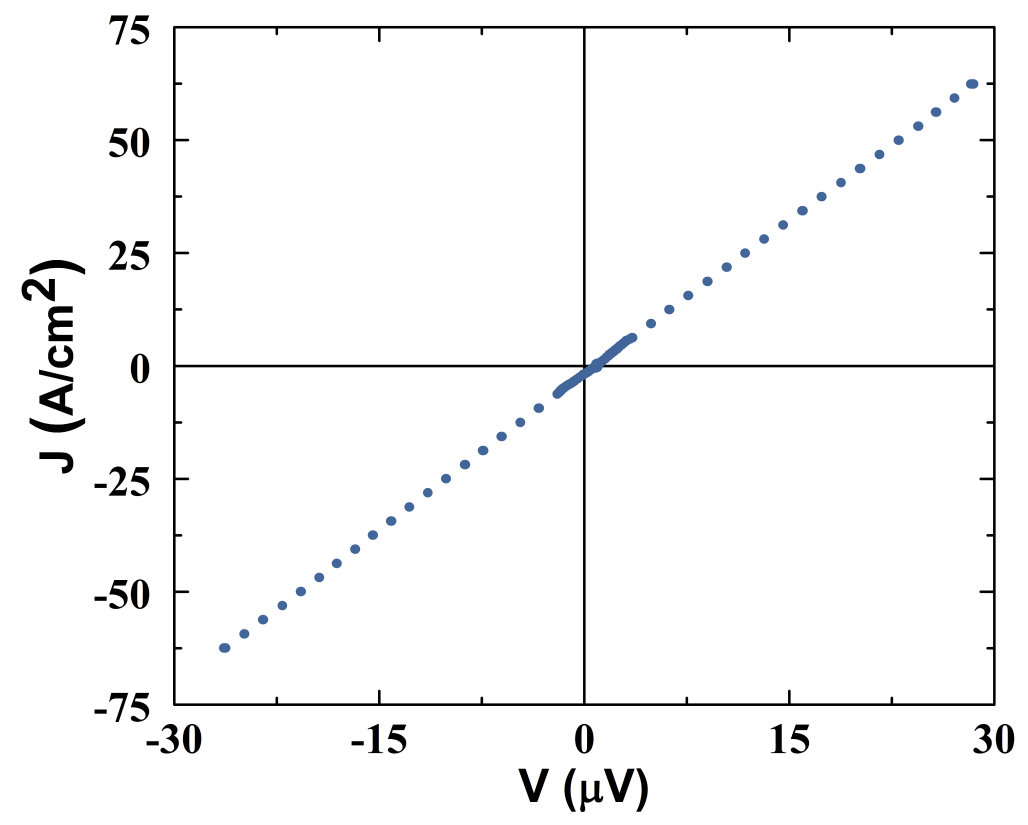

Figure 3.3: JV characteristics of an $\mathrm{Au} / \mathrm{GdN} / \mathrm{Al}$ structure, measured in a CPP geometry at $4 \mathrm{~K}$. 
junctions[49]. Because the UHV system has only 4 sources available for evaporation, Au was not used as a metallic capping layer for GdN. However, depositing $\mathrm{Cr} / \mathrm{Au}$ for use as a bottom contact using an Angstrom engineering Nexdep evaporator allowed the characterisation of the contact, as $\mathrm{Al}$ could be used as a ohmic top contact and any non-ohmic behaviour could be attributed to the $\mathrm{Au} / \mathrm{GdN}$ interface.

Figure 3.3 shows the JV curve taken at $4 \mathrm{~K}$ for one $\mathrm{Au} / \mathrm{GdN} / \mathrm{Al}$ structure. The behaviour is clearly linear, indicating the ohmic nature of both contacts. The resistivity of the $\mathrm{GdN}$ taken from measurements of $\mathrm{Au} / \mathrm{GdN} / \mathrm{Al}$ structures is $\sim 3 \Omega . \mathrm{cm}$, consistent with $\mathrm{Al} / \mathrm{GdN} / \mathrm{Al}$ structures. From these observations we conclude that both $\mathrm{Al}$ and $\mathrm{Au}$ form ohmic contacts to GdN.

\subsection{GdN with Gd contacts}

The assessment of $\mathrm{Gd}$ metal as a possible contact material is important as the exchange interaction with GdN across the material interface provides the pinning of the GdN magnetisation that allows observation of switching behaviour of the MTJ. Similarly to the the case of aluminium, a structure comprising a single $72 \mathrm{~nm}$ thick GdN layer with top and bottom contacts of $75 \mathrm{~nm}$ of $\mathrm{Gd}$ metal was deposited on $\mathrm{SiO}_{2}$ and measured to establish the electrical characteristics of the contact interfaces.

As can be seen in Figure 3.4, the IV plot of a sample with Gd metal contacts is linear at ambient temperatures but contains a slight non-linearity at low temperature. This is represented also in the temperature-dependence plot of $\mathrm{V} / \mathrm{J}$ for the same sample (see Figure 3.5). This non-linearity of the low-temperature JV curve is an indication of a differing mode of electrical transport across the interfaces of the structure. This is supported by the derivation of $\mathrm{V} / \mathrm{J}$ from the measured temperature-dependent resistance. The Gd-contacted GdN gives a value of $5 \mathrm{~m} \Omega . \mathrm{cm}^{2}$ at $4 \mathrm{~K}$, an increase by a factor of $10^{2}$ on the $32 \mu \Omega . \mathrm{cm}^{2}$ of the Al-contacted GdN. One would expect 


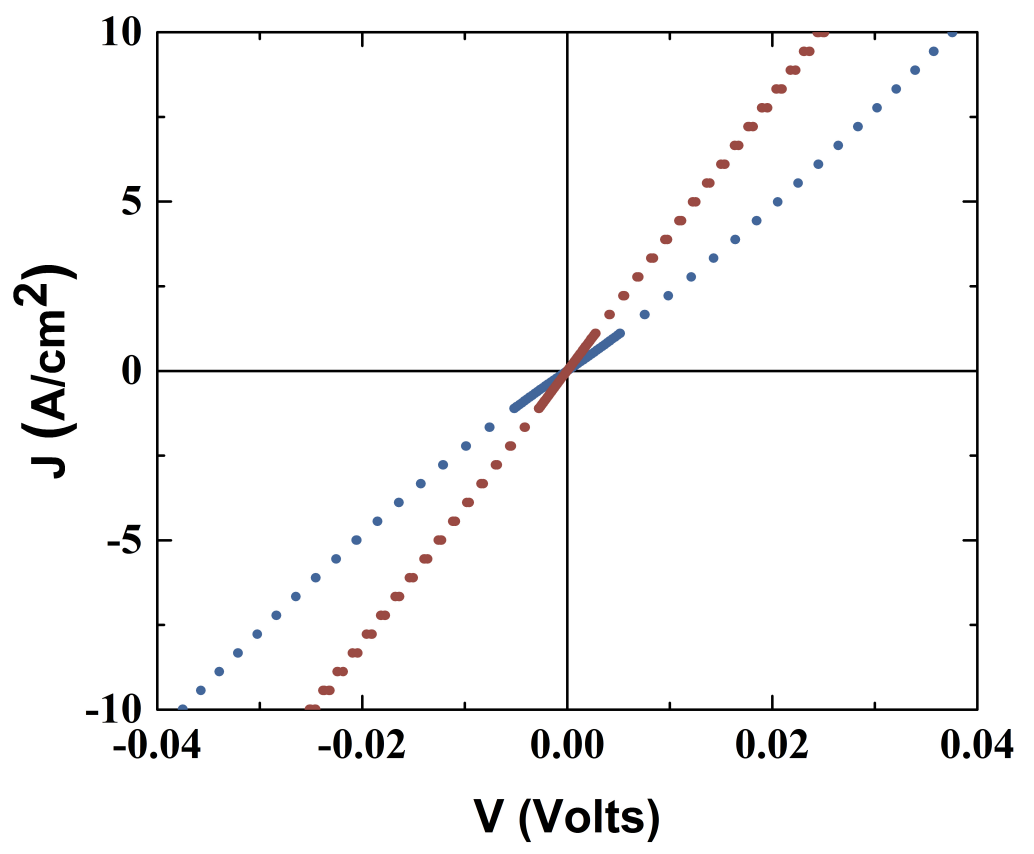

Figure 3.4: JV characteristics of GdN in a CPP geometry with Gd contacts. The blue scatter points indicate data taken at $4 \mathrm{~K}$ while the red were taken at $300 \mathrm{~K}$.

similar values of the resistance-area product of the structures, for contacts made of reasonably conductive metals, if the conduction across the interface was ohmic in each case. From the resistivity of GdN measured in the Al contacted sample $(\sim 3 \Omega . \mathrm{cm})$, it is possible to approximate the contribution of the GdN layer to V/J. Doing so in equation 3.3

$$
\frac{V}{J}=R A=\frac{\rho t}{A} \times A=23 \mu \Omega \cdot \mathrm{cm}^{2}
$$

gives a value which is not near enough to account for the much larger value calculated from the experimental data. This strongly supports a model of charge conduction dominated by GdN/Gd interface effects.

Deriving the GdN resistivity by assuming the conduction of GdN dominates the transport lends further support to this conclusion. From lowtemperature data at low applied bias one derives a resistivity of $\sim 500 \Omega . \mathrm{cm}$, 


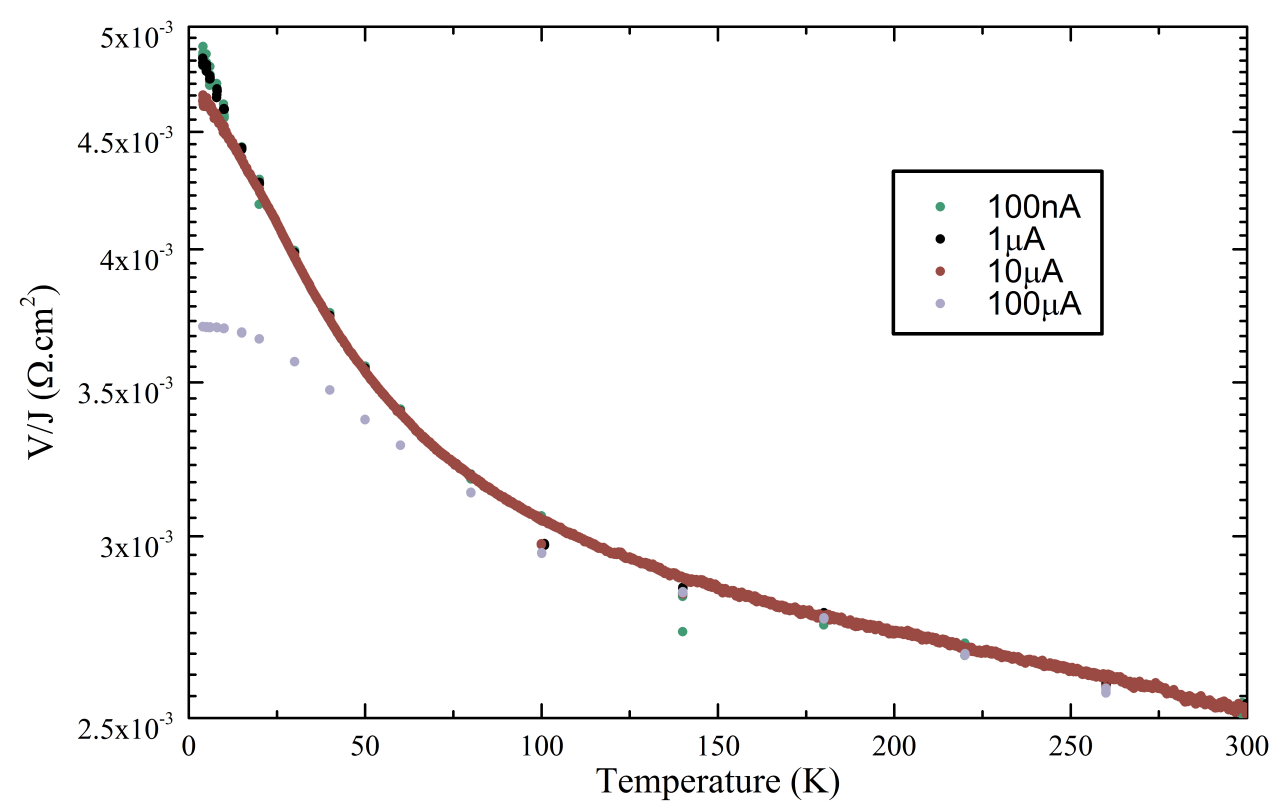

Figure 3.5: Temperature dependence of V/J for GdN between two Gd contacts. Different colour scatter points indicate different currents used to probe the response.

a value much higher than otherwise seen for GdN samples (in CPP or CIP geometries) and this, coupled with the non-linearity of the IV curve, indicates that measurements are no longer dominated by purely resistive transport through the GdN layer. Furthermore, performing the same analysis on data recorded at ambient temperature suggests a resistivity of $\sim 350 \Omega$.cm which, being much larger than typical resistivities of $\mathrm{GdN}$ grown in these conditions suggests different transport modes dominate even at temperatures which do not exhibit non-linear IV curves.

The crossover from linear to non-linear transport when decreasing the temperature suggests thermal activation of carriers over some interface barrier. This behaviour is typical of Schottky barrier formation at the interface between metal and semiconductor, the mismatch of bands causing a small region of the semiconductor to be depleted of electrons. This de- 
pletion layer would also affect the transport properties at temperatures where there is no perceived non-linearity in the IV curves. The depletion described by the Schottky barrier model is a result of the removal of charge carriers from the $\mathrm{GdN}$, an effect that would cause an increase in the resistivity of GdN even at ambient temperatures, as observed above.

\subsection{MTJs with metal contacts}

The introduction of a GaN tunnel barrier inbetween two GdN layers constitutes the production of a magnetic tunnel junction between the two metal contacts. Here, the aluminium is used to provide an ohmic bottom contact to the device. While Gd does not appear to form a similar ohmic contact to GdN it does have the benefit of pinning the GdN magnetisation by way of the ferromagnetic exchange interaction when a magnetic field is applied. This effect allows the observation of switching of the device magnetoresistance (seen in the following chapter).

The IV curves shown in Figure 3.6 exhibit a strong non-linearity, as expected for a structure whose transport is dominated by tunnelling through a wide band-gap insulator like GaN. Electrical measurements performed on an MTJ in the CPP geometry effectively treat each layer of the film as a series resistance contributing to the total resistance of the device. The electrical characteristics of the structure are dominated by the largest series resistance included in the device, in this case, transport through GaN barrier. An example that strongly illustrates this point can be found in the voltage measurements across the structure at $4 \mathrm{~K}$, while applying a current density of $1 \mathrm{~A} / \mathrm{cm}^{2}$ (refer to Figures 3.4 and 3.6). For Gd/GdN/Gd structures we found a significant contribution to the device behaviour from interface effects and a total voltage across the sample $\sim 5 \mathrm{mV}$. For MTJs with a GaN tunnelling barrier the voltage across the device when passing a current density of $1 \mathrm{~A} / \mathrm{cm}^{2}$ was on the order of a volt at the same temperature. This strongly suggests that the GaN barrier is indeed the dominant 


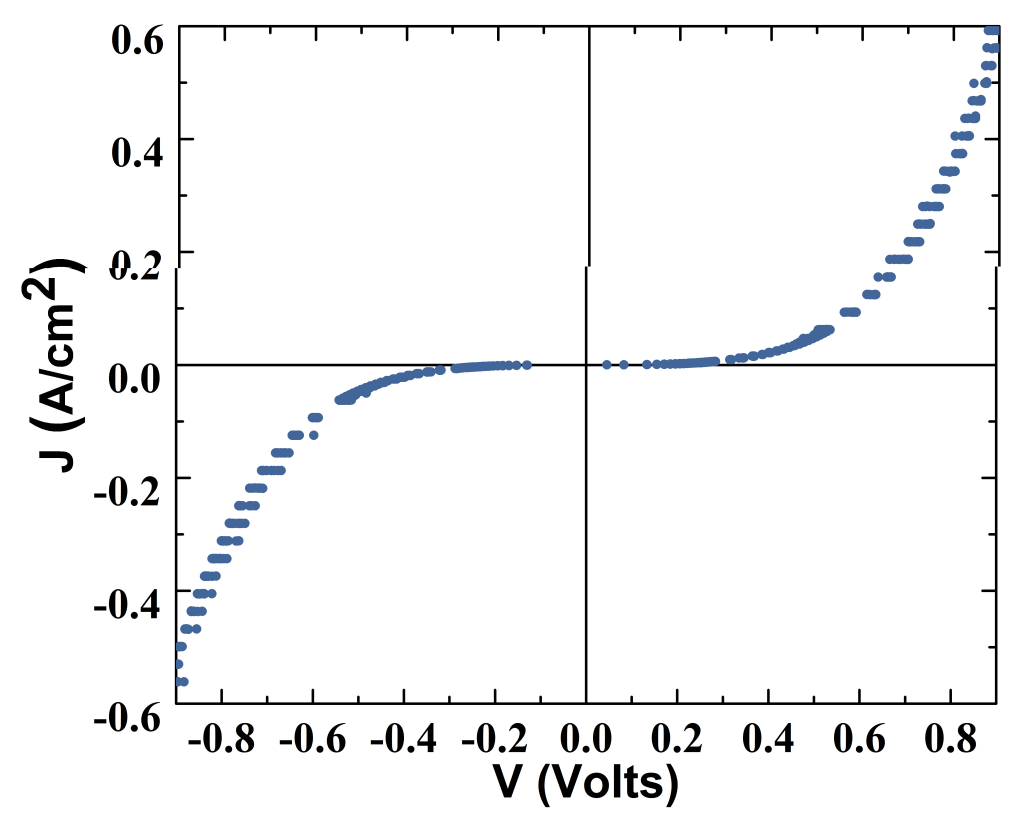

Figure 3.6: JV curves of a magnetic tunnel junction with $\mathrm{Al}$ and $\mathrm{Gd}$ as top and bottom contact respectively. Data taken at $4 \mathrm{~K}$

resistance in the device structure.

Figure 3.7 shows the increase in $\mathrm{V} / \mathrm{J}$ that results from the inclusion of the tunnel barrier. Calculating V/J for the MTJ device gives a value of $9.2 \Omega . \mathrm{cm}^{2}$, a factor of $\sim 10^{3}$ greater than the case of $\mathrm{Gd} / \mathrm{GdN} / \mathrm{Gd}$ structures and $>10^{6}$ times greater than $\mathrm{Al} / \mathrm{GdN} / \mathrm{Al}$. Clearly, a major change to the electrical transport through the stack occurs upon the introduction of the GaN barrier. To quantitatively assay the parameters that govern the barrier contribution to the transport properties, the model of electron tunnelling derived by Simmons is used. 


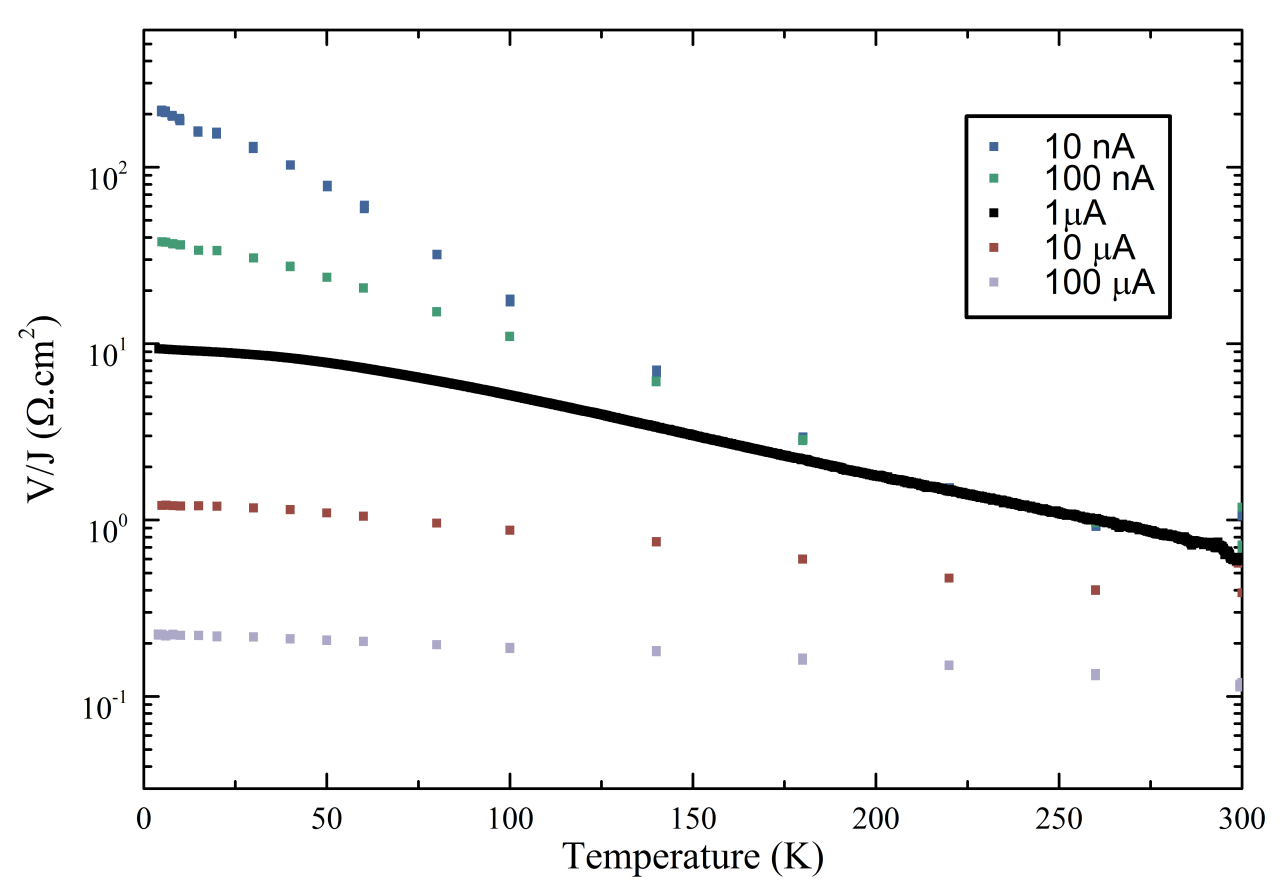

Figure 3.7: Temperature dependence of V/J for an MTJ with Al bottom contact and Gd top contact. Different colour scatter points indicate different currents used to probe the response.

\subsection{Discussion}

The dominance of the tunnelling barrier over the device electrical characteristics is shown beyond a doubt by the comparison of $\mathrm{V} / \mathrm{J}$ for device structures with and without GaN introduced. From the perspective of intended memory device functionality, the inconsequence of series resistances that result from transport through the GdN layers and across the metal/GdN interfaces is a positive result. As discussed in the introduction to Schottky interface behaviour, metal/semiconductor interfaces are notorious for the formation of barriers to electron conduction. However, in the case of $\mathrm{Gd}$ and $\mathrm{Al}$ contact metals the effect of interface behaviour 
on conduction can be actively ignored for samples with insulating barrier layers.

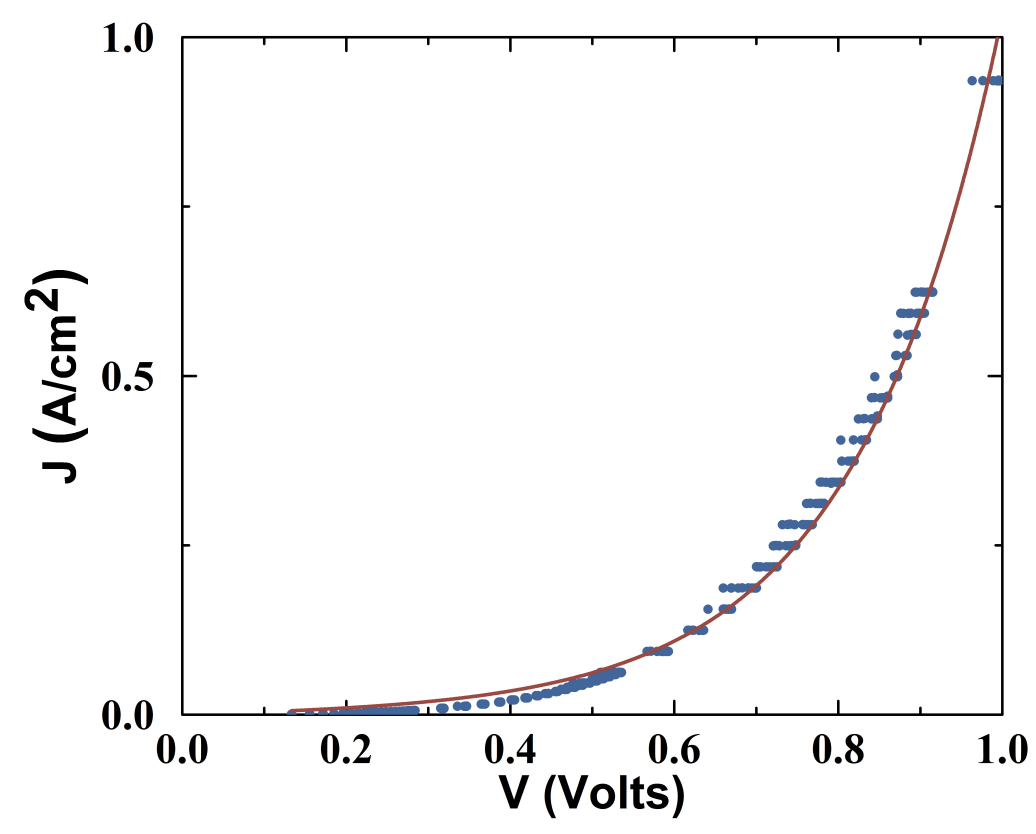

Figure 3.8: Fit of the Simmons model to the JV curve shown in Figure 3.6.

Using the Simmons model to describe MTJ devices provides a way to assess the properties of the barrier layer that determine its electrical response. The model was fit to the data in the software Origin (version 9.1) using a least squares fitting method. Performing this fit for the MTJs between $\mathrm{Al}$ and $\mathrm{Gd}$ contacts provided an estimate of $\Delta s=2.5 \mathrm{~nm}$ for the width of the GaN. The value derived from the fit agreed with that suggested by in-situ monitoring of the growth thickness by a quartz crystal microbalance $(2.8 \mathrm{~nm})$. In fact, the barrier width taken from the Simmons model tends to underestimate the width of realistic tunnelling barriers. Polycrystalline barriers like those in this project have a thickness that may be thinner or thicker as a result of the contact surface morphology. Because 
the tunnelling current induced by a given voltage is related exponentially to the barrier thickness, the parts of the barrier that dominate the electrical conductance (and thus, those that contribute most to the data fit by the model) will be those that are the thinnest. In this case, the Simmons model will return an average barrier width that may be somewhat smaller than estimates of the barrier width provided by other means.

The Simmons model also returned a value of $\varphi_{I}=1.4 \mathrm{eV}$ for the height of the potential barrier. This fits with the estimate of the potential barrier height that comes from the band structures of the device materials. As GdN has a small band gap and is doped by nitrogen vacancies, its Fermi level lies somewhere in the vicinity of the conduction band edge (perhaps slightly below). In contrast, $\mathrm{GaN}$ is a wide band gap semiconductor with a gap $\sim 3.4 \mathrm{eV}$. As the Fermi levels align across the interface between the materials, one would expect the barrier height provided by the Simmons model to be half of the total band gap of $\mathrm{GaN}$ to a first approximation. Such an approximation appears to be valid here.

These results suggests that the control of the electrical characteristics of GdN-based MTJs by the GaN tunnel barrier is strong. The efficacy of a simple Simmons model in describing the conduction is effective and this is a positive result from the point of view of development of applications. 


\section{Chapter 4}

\section{GdN Structures with Superconducting Contacts}

This chapter concerns the integration of REN-structures with niobium $(\mathrm{Nb})$, the material typically used for superconducting electronics. Chapter 2 included assessment of the physical compatibility of the RENs with $\mathrm{Nb}$ by means of AFM, SEM and XRD. This chapter details the electrical characteristics of devices measured in a CPP geometry.

\subsection{GdN with $\mathrm{Nb}$ contacts}

The nature of the contact between $\mathrm{Nb}$ and $\mathrm{GdN}$ was investigated in electrical measurements through a $\mathrm{Nb} / \mathrm{GdN} / \mathrm{Nb}$ structure. The samples were electrically characterised by taking IV curves and determining the JV characteristics and temperature dependence of V/J. Figure 4.1 shows the JV curves for these samples derived assuming the area is the nominal area of the device (measurements taken at $4 \mathrm{~K}$ ). It is clear from the non-linearity of the curves that the conduction is not ohmic.

Another feature of the $\mathrm{Nb}$-contacted GdN samples is the marked departure from a geometry-independent relationship between current density and voltage, similar to the samples grown with Al contacts. In Figure 


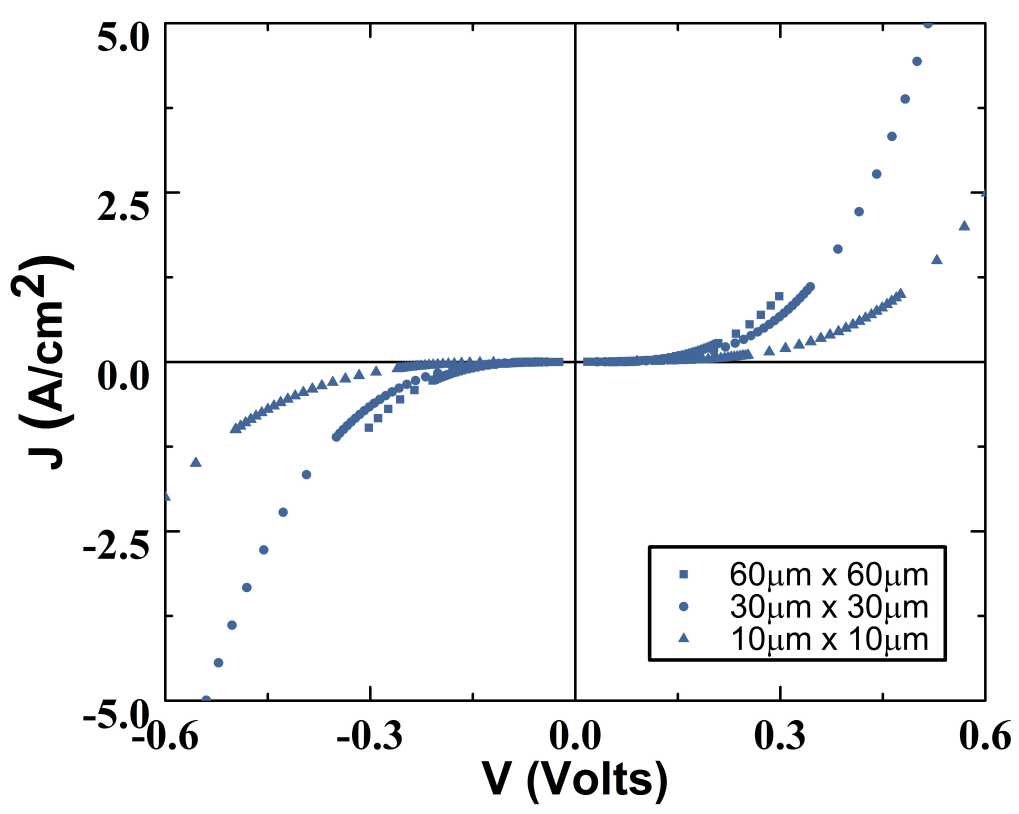

Figure 4.1: JV curves of GdN in a CPP geometry with $\mathrm{Nb}$ contacts. All data were taken at $4 \mathrm{~K}$. The different scatter point shapes indicate different junction sizes.

4.1 the shapes of the different scatter points indicate the size of the device measured (nominally $60 \times 60,30 \times 30$ or $10 \times 10 \mu \mathrm{m}$ ). In order to account for the failure of the current to scale with the area we explored the assumption that part of the device area was non-conductive, as we did in the case of $\mathrm{Al}$ contacts. By plotting the data together and making a subtraction of 6 microns from the device side length (the same as with $\mathrm{Al}$ contacts) the JV curves were made to overlay. This gives the single current density induced by a given voltage independent of the device size, shown in Figure 4.2 .

The origin of the non-linearity in the $\mathrm{JV}$ plots for $\mathrm{Nb}$ contacted samples is not made clear by this plot. When conducting electrical measurements of the samples with metallic contacts we found non-linearity resultant from both insulating barriers in the sample and Schottky-like contacts 


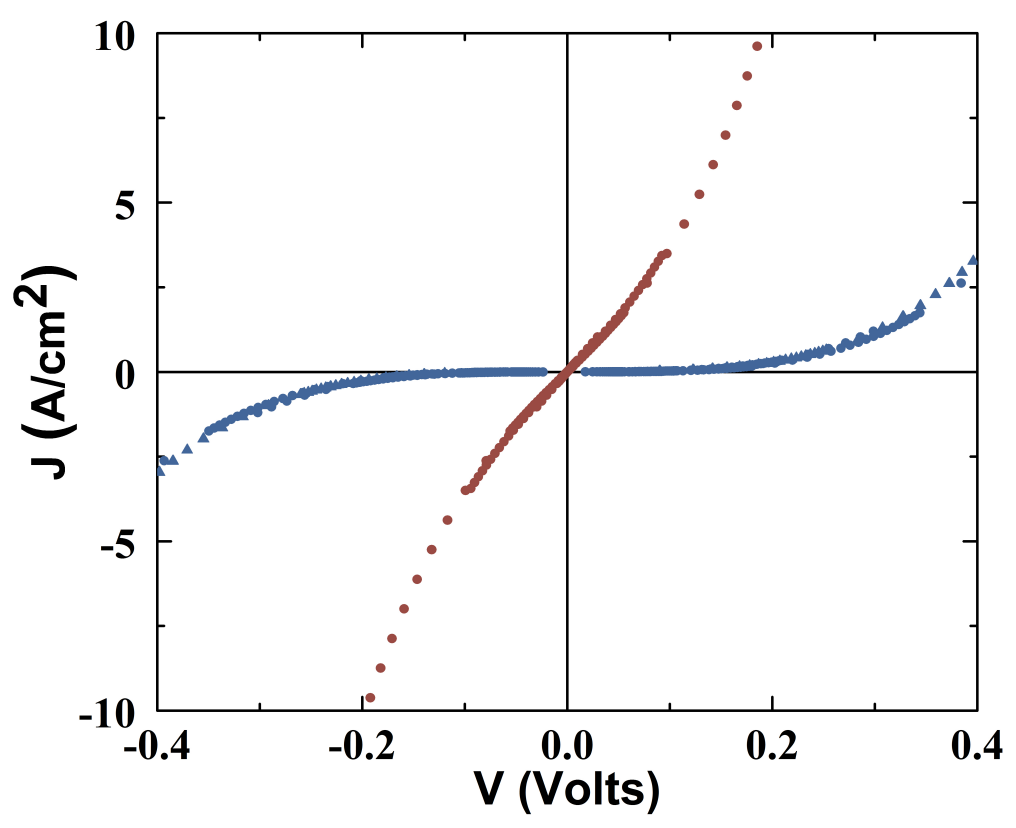

Figure 4.2: JV curves of GdN in a CPP geometry with $\mathrm{Nb}$ contacts. The blue scatter points indicate data taken at $4 \mathrm{~K}$, red at $300 \mathrm{~K}$. The different scatter point shapes indicate different junction sizes.

at interfaces. It is worth noting that the plot of the temperature dependence of $\mathrm{V} / \mathrm{J}$ for such samples is similar to that of the samples with $\mathrm{GaN}$ introduced as a barrier, and one might expect that the non-linearity for the $\mathrm{Nb}$-contacted samples is a result of a similar insulating barrier, such as an oxide formed at a metal interface. Because the device fabrication involves 3 steps in which the samples are processed lithographically using various solvents, it is plausible that such an oxide barrier is formed at the surface of the $\mathrm{Nb}$ bottom contact when the chip is exposed to air, though this raises the question of why we do not see a similar non-linear curve for GdN contacted with $\mathrm{Al}$ or Gd.

The temperature-dependence of the resistance-area product is shown in 4.3. The non-linearity of the JV plot can be observed on this plot as 


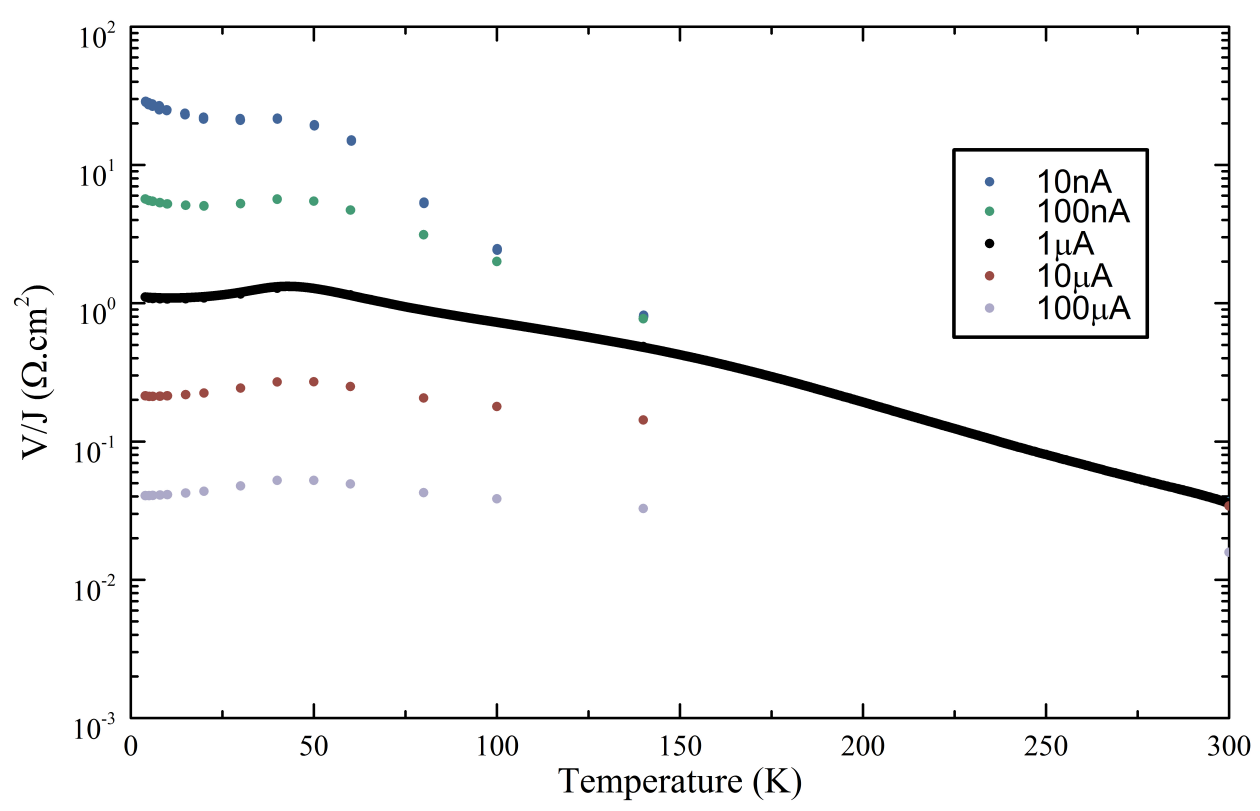

Figure 4.3: Temperature dependence of the $\mathrm{V} / \mathrm{J}$ for GdN between two $\mathrm{Nb}$ contacts. Different colour scatter points indicate different currents used to probe the response.

the strong differences in $\mathrm{V} / \mathrm{J}$ for different applied currents (indicated by the differently coloured scatter points). A typical operation current of $1 \mu \mathrm{A}$ returns a $\mathrm{V} / \mathrm{J}$ value of $\sim 1 \Omega . \mathrm{cm}^{2}$ at $4 \mathrm{~K}$.

In order to explore the nature of the contacts further, samples were grown with one $\mathrm{Nb}$ contact opposite an ohmic contact. One such sample was a $\mathrm{Au} / \mathrm{GdN} / \mathrm{Nb}$ structure with Au bottom and $\mathrm{Nb}$ top contact. Figure 4.4 shows the low temperature JV curves from such a sample. Because gold forms an ohmic contact to GdN (see Figure 3.3), the non-linearity of the JV curves for this sample suggest Schottky contact at the interface between the GdN and $\mathrm{Nb}$. An oxide barrier is exceedingly unlikely to form at the $\mathrm{GdN} / \mathrm{Nb}$ interface as the sample is not removed from the UHV chamber between these two growth steps, and the residual partial pressure of water (a likely culprit for oxidation in this context) in the growth 
chamber is $10^{-9}$ mbar. The JV curve shown in Figure 4.4 shows pronounced asymmetry. Such asymmetry is a feature commonly exploited in devices such as Schottky diodes, and its appearance in a sample of this form constitutes strong evidence of Schottky contact formation at the $\mathrm{GdN} / \mathrm{Nb}$ interface.

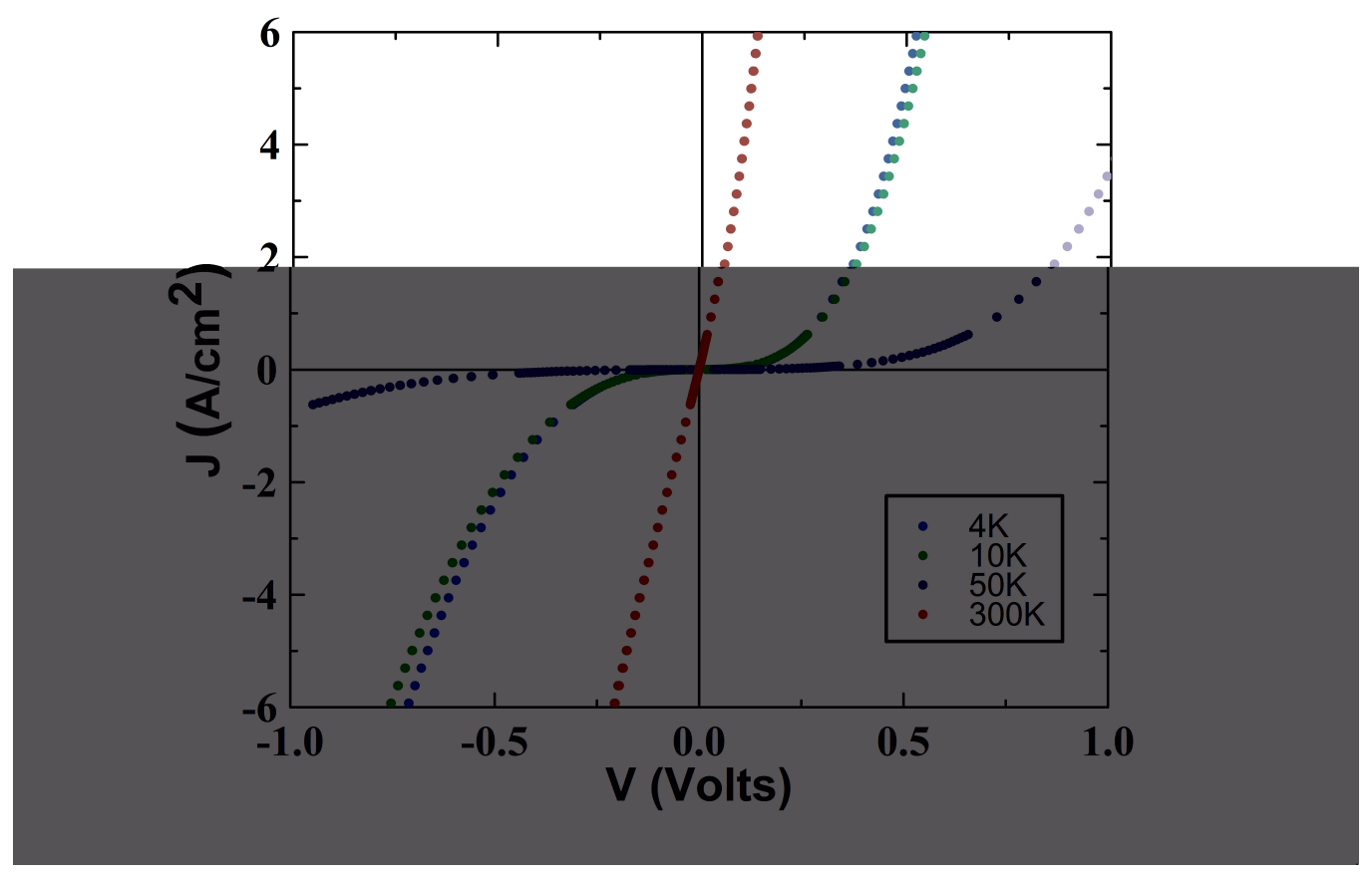

Figure 4.4: JV curves of GdN in a CPP geometry with Au-bottom and Nb-top contacts. The blue scatter points indicate data taken at $4 \mathrm{~K}$, green were taken at $10 \mathrm{~K}$, purple at $50 \mathrm{~K}$ and red at $300 \mathrm{~K}$.

A plot showing the temperature dependence of $\mathrm{V} / \mathrm{J}$ of this $\mathrm{Au} / \mathrm{GdN} / \mathrm{Nb}$ structure is displayed in Figure 4.5. The strong non-linearity of the JV curves is clear in this representation of the electrical data. The contribution of the GdN to some aspect of the conduction is suggested by the change in behaviour that occurs around the Curie temperature of GdN, however it is not clear that this is a manifestation of transport through the GdN layer or an interface effect. $\mathrm{V} / \mathrm{J}$ is $\sim 3 \Omega . \mathrm{cm}^{2}$ for an operating current of $1 \mu \mathrm{A}$. 
This is a factor of $10^{5}$ larger than the $\mathrm{Al} / \mathrm{GdN} / \mathrm{Al}$ structures presented in Figure 3.2, an indication of the huge effect of some form of barrier on the electron transport.

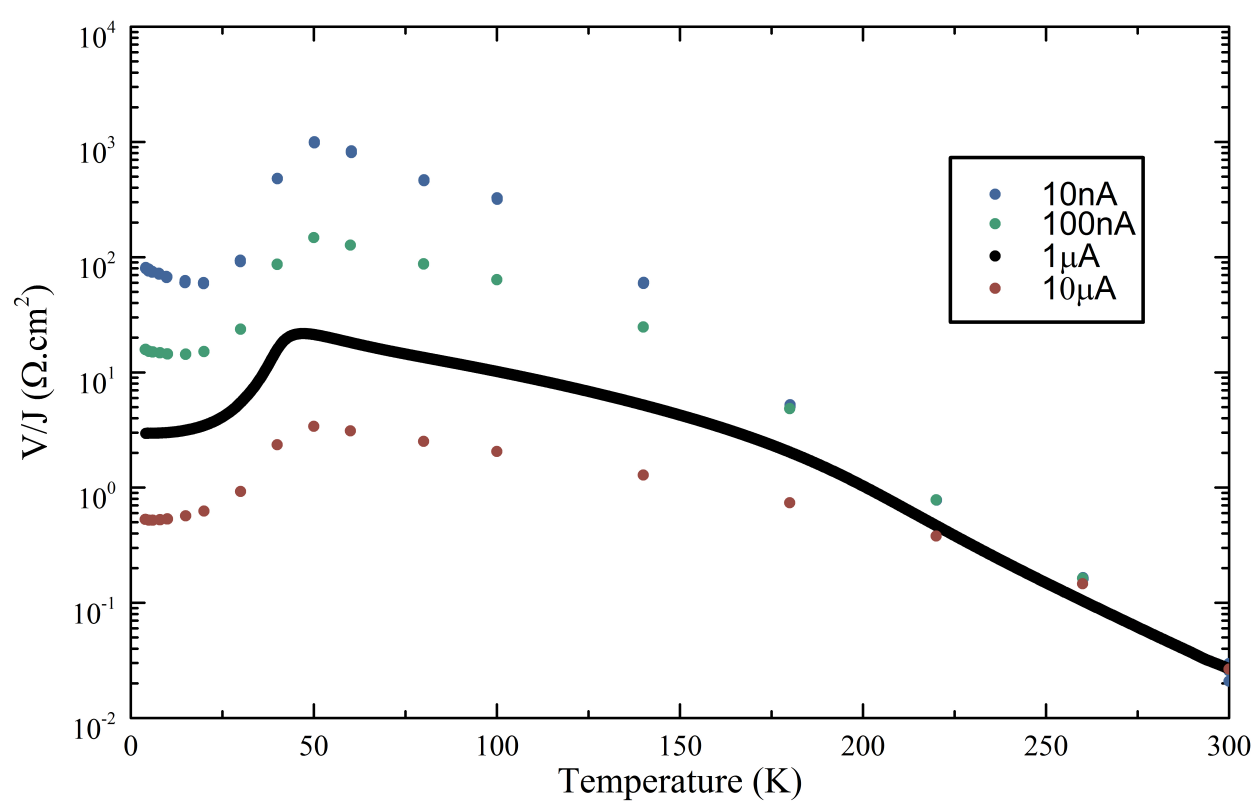

Figure 4.5: Temperature dependence of $\mathrm{V} / \mathrm{J}$ for $\mathrm{GdN}$ between a Au-bottom and $\mathrm{Nb}$ top contact. Different colour scatter points indicate different currents used to probe the response.

A second sample was grown with one ohmic and one superconducting contact to observe the contact behaviour, but in the opposite orientation. This sample had a $\mathrm{Nb}$ bottom contact and was capped with an Al top contact, the temperature dependence of $\mathrm{V} / \mathrm{J}$ is shown in 4.6. This sample, with $\mathrm{Nb}$ bottom contact and $\mathrm{Al}$ top, exhibits a fairly strong non-linearity at low temperature, but appears to have ohmic contact between interfaces when measured at ambient temperature. Such behaviour may be indicative of thermal activation over some Schottky barrier. $\mathrm{V} / \mathrm{J}$ is a factor of 100 smaller than in the case of the $\mathrm{Au} / \mathrm{GdN} / \mathrm{Nb}$ samples (those with $\mathrm{Nb}$ 
as a top contact), and there is no obvious change in the electrical transport upon the magnetic transition of the GdN (this may not be true for high applied biases, but the magnitude of the effect is much smaller than in Figures 4.3 and 4.5). This is a marked contrast with the $\mathrm{Au} / \mathrm{GdN} / \mathrm{Nb}$ samples, which saw a clear change in the transport behaviour (particularly a strong reduction of the JV asymmetry) below the magnetic transition temperature of $\mathrm{GdN}$. These results imply a correlation, between these effects and the use of $\mathrm{Nb}$ to provide the $\mathrm{GdN}$ top-contact.

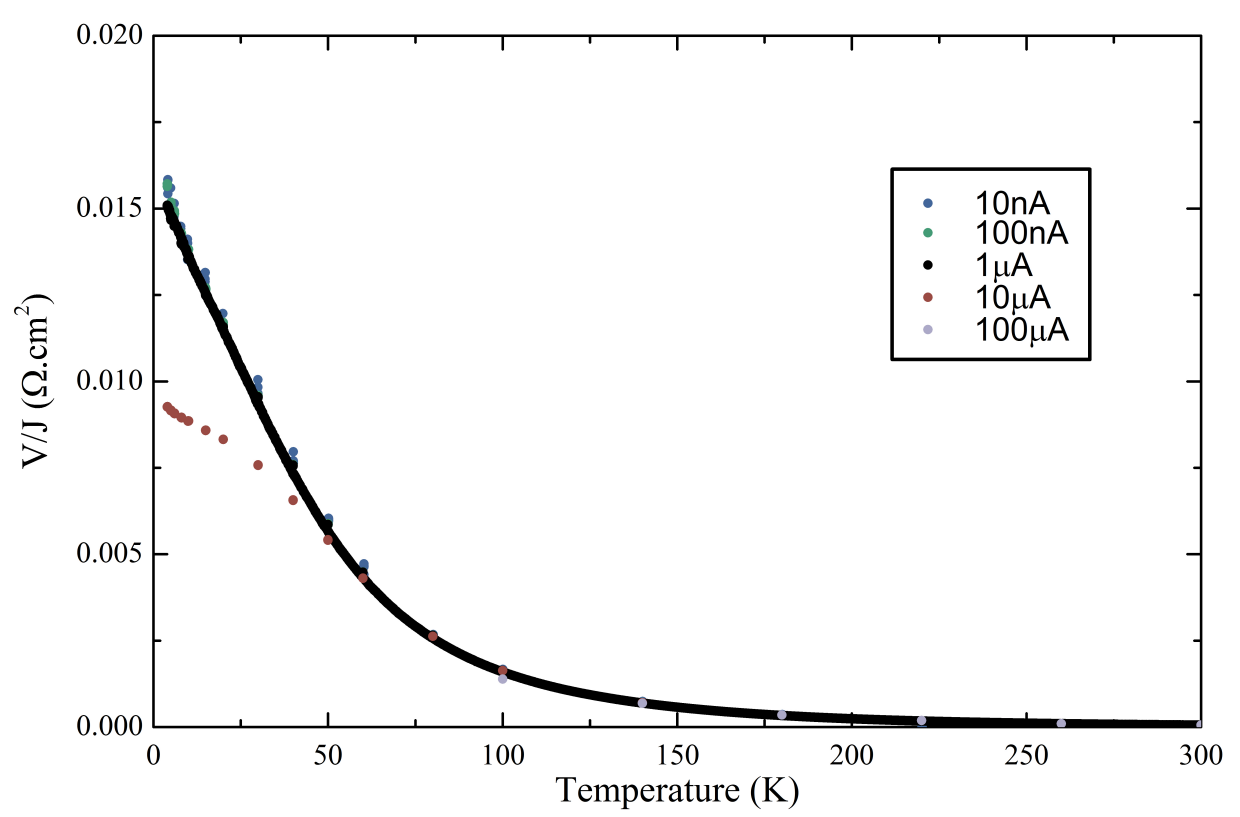

Figure 4.6: Temperature dependence of $\mathrm{V} / \mathrm{J}$ for GdN in a CPP geometry with $\mathrm{Nb}$ bottom and Al-top contacts. The colours of the scatter points indicate the different currents used for the measurements. 


\subsection{MTJs with $\mathrm{Nb}$ contacts}

This section details attempts to electrically characterise MTJs grown with $\mathrm{Nb}$ contacts. Determination of $\mathrm{V} / \mathrm{J}$ from measurements made on MTJs with $\mathrm{Al}$ and $\mathrm{Gd}$ contacts (presented in Chapter 3), found values on the order of $\sim 10 \Omega . \mathrm{cm}^{2}$ for an operating current of $1 \mu \mathrm{A}$. Such large values indicate that the insulating GaN barrier in the sample dominates the electrical transport in the device. The previous section presented measurements of GdN in a CPP geometry using $\mathrm{Nb}$ contacts. These measurements exhibit strong contributions of interface effects to the electron transport and values of $\mathrm{V} / \mathrm{J}$ as high as $3 \Omega . \mathrm{cm}^{2}$ were derived from measurements.

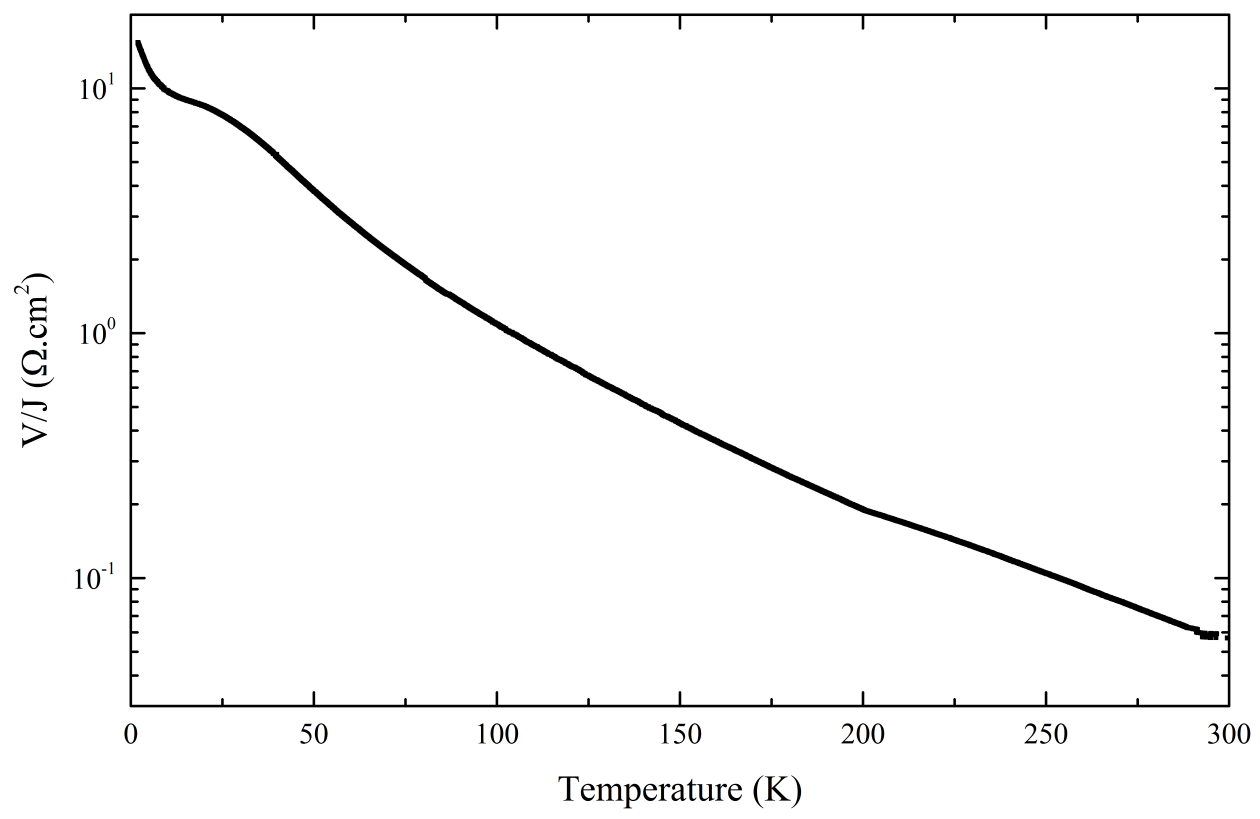

Figure 4.7: Temperature dependence of V/J for a GdN-based MTJ with a Nb bottom contact. Operating current $=1 \mu \mathrm{A}$

Figure 4.7 shows the temperature-dependence of V/J for an MTJ with $\mathrm{Gd}$ and $\mathrm{Nb}$ thickness of $90 \mathrm{~nm}$ and GdN electrodes $120 \mathrm{~nm}$ thick. This sam- 
ple exhibits similar V/J characteristics to the MTJ presented in Chapter 3 (Figure 3.7). The effect of the introduction of $\mathrm{GaN}$ appears to be consistent across devices with dissimilar contact materials, with $\mathrm{V} / \mathrm{J}>10 \Omega . \mathrm{cm}^{2}$ at low temperature and an applied current of $1 \mu \mathrm{A}$.

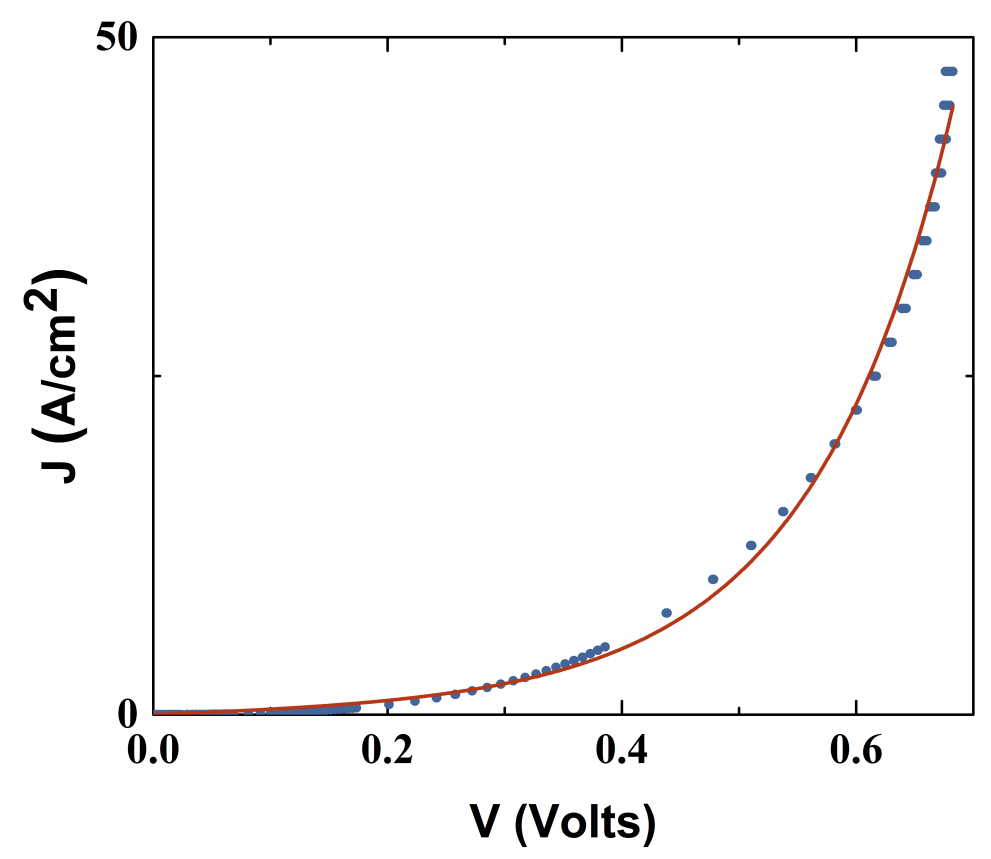

Figure 4.8: Simmons model fit to the JV curve of an MTJ with Nb contacts. Data taken at $4 \mathrm{~K}$.

The relationship between voltage and current density is plotted in Figure 4.8 with a Simmons model fitted to the data. As with the MTJ data presented in Chapter 3 it is possible to extract an estimate of the potential barrier height and width using this model. However, the fit sees less success in this case. The model consistently gives $2.3 \mathrm{~nm}$ as the barrier width, slightly smaller $(<1 \mathrm{~nm})$ than the thickness expected from calibration growth rates, as in the case of MTJs with $\mathrm{Al}$ and Gd contacts. Yet, the barrier height produced by the model had a large range of uncertainty and varied significantly across different temperatures. 


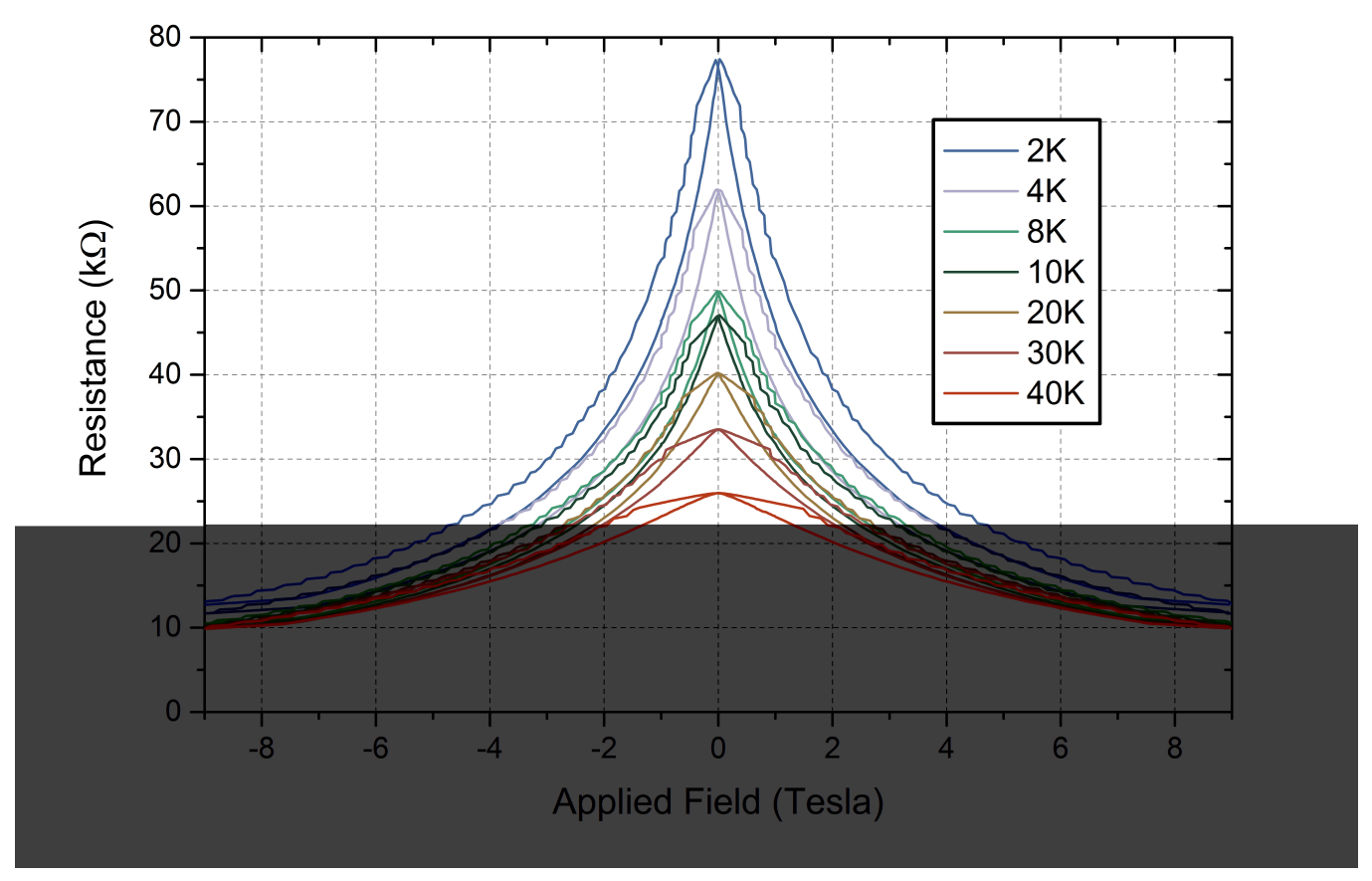

Figure 4.9: Magnetoresistance of a GdN-based MTJ with superconducting Nb bottom contact, measured at an operating current of $1 \mu \mathrm{A}$

Finally, V/J was measured for the same sample as a function of magnetic field (swept from 9 Tesla to -9 Tesla and back) in order to observe whether the behaviour of the devices was characteristic of an MTJ. The hysteresis that appears can be attributed to the magnetisation pinning of the top electrode by the Gd top contact, giving the high resistance state. The result is plotted in 4.9. Immediately clear from the plot is the strong magnetic response of the sample. A switching effect that results from the changing magnetisation is observed at low applied fields. Using equation 1.1

$$
M R=\frac{R_{A P}-R_{P}}{R_{P}}
$$

to calculate the tunnelling magnetoresistance of the sample returns a value $>500 \%$ (here $R_{A P}$ and $R_{P}$ are taken as the maximum and minimum of 
the magnetoresistance scan). This is an unprecedentedly high value for an unoptimised polycrystalline junction, with values in the literature for GdN-based electrodes being 200\%[33].

\subsection{Discussion}

The failure of the Simmons' model to describe the tunnelling barrier of the MTJ with $\mathrm{Nb}$ contacts is a strong indication of interference from some series resistance that is part of the device structure. The switching at low fields is characteristic of MTJ structures. However, the typical switching effect is imposed on a strong secondary effect that doesn't appear to saturate even out to 9T. One result that can potentially be attributed to this secondary contribution to the magnetoresistance is a high TMR value. GdNbased MTJs grown in the past on metals such as gold have had TMR values of $\sim 200 \%$ [33]. Though the mechanism of the effect is not clear at this stage, the values quoted in this chapter provide a significant improvement on previous GdN-electrode MTJs and understanding this mechanism provides a clear direction for further research.

An interesting manifestation of the electrodynamics of the magnetoresistance measurement is the strong shape anisotropy evident in the measurement. Figure 4.9 shows the magnetoresistance at a variety of temperatures when the magnetic field is applied in the plane of the thin film. The data taken at $2 \mathrm{~K}$ are plotted again in Figure 4.10 in blue, with a second trace in red showing the same measurement made when the magnetic field was applied normal to the plane of the film. This plot indicates a strong shape anisotropy with the function of $\mathrm{R}(\mathrm{B})$ broadened significantly for measurements made with the applied field normal to the plane of the film. The broadening that results also leads to a more visible switching of the magnetic domains in the sample.

Directions for further research can be motivated by the application of the Julliére model of TMR referenced in Chapter 1. This model relates the 


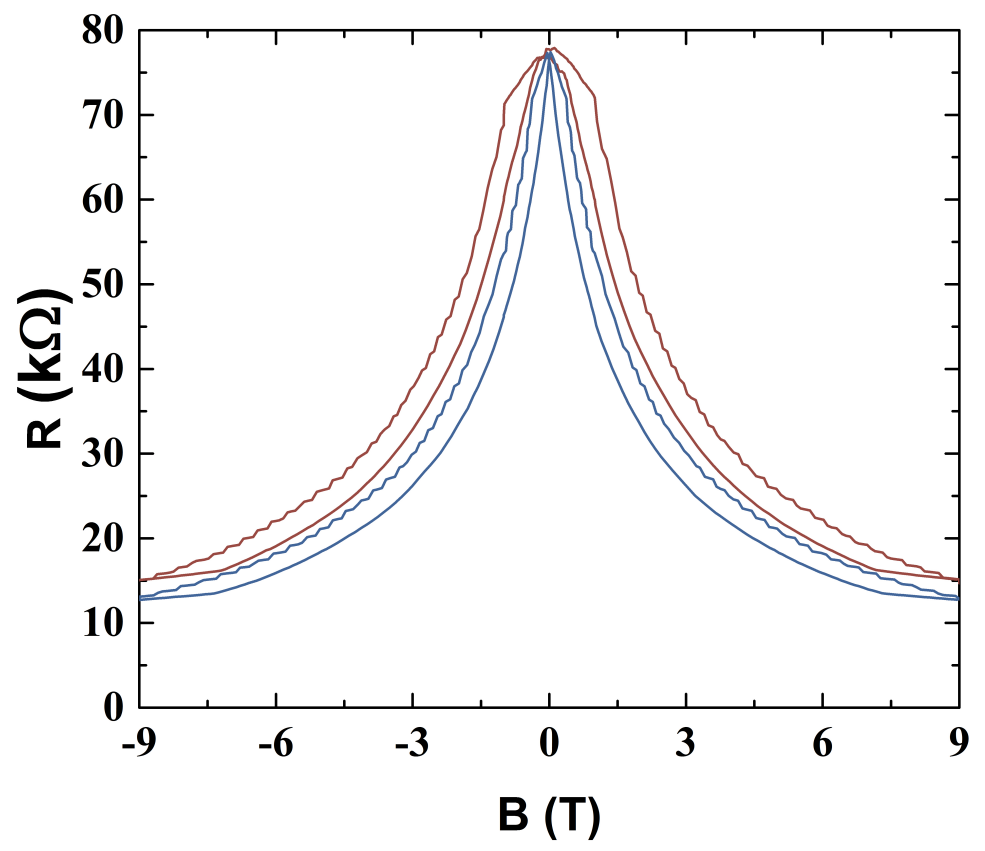

Figure 4.10: Magnetoresistance of a GdN-based MTJ with superconducting Nb bottom contact, measured at an operating current of $1 \mu \mathrm{A}$ and at $2 \mathrm{~K}$. The blue trace represents data taken when the magnetic field was applied in-plane, the red indicates the application of the magnetic field in the direction normal to the film plane.

TMR quantitatively to strong spin polarisations in the electrodes and was given in equation 1.2

$$
T M R=\frac{2 P_{L} P_{R}}{1-P_{L} P_{R}} .
$$

The model is the first used to describe the TMR effect (introduced in 1975) and fails to account for the roughness of the interfaces and other experimental considerations. Furthermore, the application of Julliere's model is almost certain not to describe the tunnelling in the devices presented in this thesis, afflicted as they are by strong interface effects. Despite this, the model provides a starting point for estimations of the real value of the spin polarisations of the electrodes. For the case of symmetric electrodes 
we make the assumption that $P_{L}=P_{R}$ and this leads to a relation

$$
T M R=\frac{2 P_{G d N}^{2}}{1-P_{G d N}^{2}}
$$

between the TMR and the spin-polarisation, returning a value of $57.7 \%$ for the spin polarisation of the electrodes. This is significantly smaller than theoretical predictions of the spin-polarisation in GdN (which approach full spin-polarisation in the low-temperature limit) and smaller than that measured in spin filter experiments in reference [50]. That said, this estimate is larger than the spin-polarisations often quoted for ferromagnetic transition metals[6]. The spin polarisation could be probed more directly by measurements of superconducting tunnelling currents into ferromagnetic GdN as in work published by Tedrow and Merservey, [51] and accurate determination of this result would be important for the technological application of the RENs. 
66CHAPTER 4. GDN STRUCTURES WITHSUPERCONDUCTING CONTACTS 


\section{Chapter 5}

\section{Conclusions}

GdN-based device structures were grown by physical vapour deposition and electrically characterised. Electrical measurements of the GdN films were initially difficult to interpret as the measurement geometries utilised led to a strong contribution from electrical conduction in the plane of the film. To combat this effect, I developed lithographic patterning methods and a photomask that allowed the growth of three devices per chip in an architecture appropriate for measurements perpendicular to the film plane (CPP geometry). Electrical measurements performed on the patterned samples returned resistivities within the range measured in CIP geometries and similar temperature-dependent behaviour.

Growth compatibility was assured between GdN and the metals used in this study ( $\mathrm{Al}, \mathrm{Au}, \mathrm{Gd}$ and $\mathrm{Nb}$ ) using XRD and SEM techniques. AFM and XRR were also used to assess the thickness and surface roughness of the metal depositions. The lack of segregation phenomena, delamination or secondary phases in the GdN films justified the use of $\mathrm{Al}, \mathrm{Au}, \mathrm{Gd}$ and $\mathrm{Nb}$ in the search for contact materials with desirable electrical transport properties.

To investigate the electrical compatibility of the various $\mathrm{GdN} /$ metal combinations, GdN thin films were grown with metallic contacts. While $\mathrm{Al}$ and $\mathrm{Au}$ were confirmed to make Ohmic contact with the GdN surface, 
Gd was found to exhibit Schottky-like behaviour at the GdN/Gd interface. GdN-based MTJs with GaN barriers were grown and measured in the same conditions and found to have electrical characteristics dominated by transport through the GaN tunnel barrier. This dominance of the GaN barrier over the device characteristics renders the non-Ohmic nature of the GdN/Gd contact inconsequential from the point of view of MTJ fabrication, as the interface effects do not appreciably alter the behaviour of the device.

Unlike using $\mathrm{Al}$, $\mathrm{Au}$ or $\mathrm{Gd}$ to contact $\mathrm{GdN}$, making contact with $\mathrm{Nb}$ led to strongly non-linear electrical effects. As $\mathrm{Nb}$ is the typical superconductor used for interconnects in superconducting electronics, its electrical integration with GdN (and in particular, GdN-based MTJs) was a major goal of this project. Interestingly, the strong non-linearity and asymmetry of JV curves associated with $\mathrm{Nb}$ contacts occurred only for samples in which $\mathrm{Nb}$ was deposited as a top contact. Samples that employed $\mathrm{Nb}$ as a bottom contact only (coupled with some Ohmic top contact) were found to have $\mathrm{V} / \mathrm{J} \sim 10^{-2} \Omega . \mathrm{cm}^{2}$, a factor of $10^{3}$ less than the MTJ samples (for which $\mathrm{V} / \mathrm{J}$ was on the order of $\sim 10 \Omega . \mathrm{cm}^{2}$ ). While the $\mathrm{Nb} / \mathrm{GdN}$ interface did not make a strong contribution to the voltage across the GdN-based MTJs (relative to the large effect of the GaN barrier), it may be implicated in the high tunnelling magnetoresistance measured for MTJs with $\mathrm{Nb}$ contacts. Measurements of $\mathrm{Nb}$-contacted MTJs had significantly greater magnetoresistance than published results grown on $\mathrm{Au}$ [33]. Manuscripts are being prepared for publication regarding the interesting contact behaviour and high magnetoresistance that have been recorded in this thesis. Further research will be needed to isolate the physical phenomena responsible for the multiple effects contributing to magnetoresistance measurements, but the values obtained here for unoptimised, polycrystalline junctions offer promise with an eye to future device applications and the integration of GdN-based devices with $\mathrm{Nb}$, the material of choice within superconducting electronics, is a major step towards practical memory devices. 


\section{Bibliography}

[1] G. E. Moore, "Excerpts from A Conversation with Gordon Moore: Moore's Law", [Online]. Available: ftp:/ /download.intel.com/museum/Moores_Law/VideoTranscripts, Intel, pp. 1-2, 2005, [Accessed 2018-05-23].

[2] M. M. Waldrop, "The chips are down for Moore's law", Nature, vol. 530, 2016.

[3] S. A. Wolf, D. D. Awschalom, R. A. Buhrman, J. M. Daughton, S. Von Molnár, M. L. Roukes, A. Y. Chtchelkanova, and D. M. Treger, "Spintronics: A spin-based electronics vision for the future", 2001.

[4] H. C. Ohanian, "What is spin?", Am. J. Phys., vol. 54, pp. 500-505, 1986.

[5] A. Gsponer, "What is spin?", [Online]. Available: arXiv:physics/0308027v3, 2003, [Accessed 2018-05-23].

[6] E. Y. Tsymbal and I. Zutic, Handbook of Spin Transport and Magnetism. Boca Raton, FL, USA: CRC Press, 2011.

[7] M. N. Baibich, J. M. Broto, A. Fert, F. N. Van Dau, F. Petroff, P. Etienne, G. Creuzet, A. Friederich, and J. Chazelas, "Giant Magnetoresistance of (001)Fe/(001)Cr Magnetic Superlattices", Phys. Rev. Lett., vol. 61, pp. 2472-2475, 1988. 
[8] U. Björksten and E. Huss, "The 2007 Nobel Prize in Physics - Press Release.", [Online]. Available: https: / / www.nobelprize.org/nobel_prizes/physics/laureates/2007/press.html, 2014, [Accessed 2018-05-23].

[9] P. M. Levy, S. Zhang, and A. Fert, "Electrical conductivity of magnetic multilayered structures", Phys. Rev. Lett., vol. 65, pp. 1643-1646, 1990.

[10] T. Valet and A. Fert, "Theory of the perpendicular magnetoresistance in magnetic multilayers", Phys. Rev. B, vol. 48, pp. 7099-7113, 1993.

[11] J. G. Simmons, "Generalized Formula for the Electric Tunnel Effect between Similar Electrodes Separated by a Thin Insulating Film", J. Appl. Phys., vol. 34, no. 6, pp. 1793-1803, 1963.

[12] J. G. Simmons, "Generalized thermal J-V characteristic for the electric tunnel effect", J. Appl. Phys., vol. 35, no. 9, pp. 2655-2658, 1964.

[13] M. Julliere, "Tunneling between ferromagnetic films", Phys. Lett. A, vol. 54, pp. 225-226, 1975.

[14] E. Y. Tsymbal, O. N. Mryasov, and P. R. LeClair, "Spin-dependent tunnelling in magnetic tunnel junctions", 2003.

[15] T. Dietl, "A ten-year perspective on dilute magnetic semiconductors and oxides", Nat. Mater., vol. 9, pp. 965-974, 2010.

[16] T. Dietl and H. Ohno, "Dilute ferromagnetic semiconductors: Physics and spintronic structures", Rev. Mod. Phys., vol. 86, pp. 187-251, 2014.

[17] T. Dietl, H. Ohno, F. Matsukura, J. Cibert, and D. Ferrand, "Zener model description of ferromagnetism in zinc-blende magnetic semiconductors", Science (80-. )., vol. 287, pp. 1019-1022, 2000.

[18] P. F. Xu, S. H. Nie, K. K. Meng, S. L. Wang, L. Chen, and J. H. Zhao, "Co doping enhanced giant magnetocaloric effect in Mn1-xCoxAs 
films epitaxied on GaAs (001)", Appl. Phys. Lett., vol. 97, p. 042502, 2010.

[19] A. Navarro-Quezada, W. Stefanowicz, T. Li, B. Faina, M. Rovezzi, R. T. Lechner, T. Devillers, F. D'Acapito, G. Bauer, M. Sawicki, T. Dietl, A. Bonanni, F. D'Acapito, G. Bauer, M. Sawicki, T. Dietl, and A. Bonanni, "Embedded magnetic phases in $(\mathrm{Ga}, \mathrm{Fe}) \mathrm{N}$ : Key role of growth temperature", Phys. Rev. B, vol. 81, p. 205206, 2010.

[20] C. M. Aerts, P. Strange, M. Horne, W. M. Temmerman, Z. Szotek, and A. Svane, "Half-metallic to insulating behavior of rare-earth nitrides", Phys. Rev. B, vol. 69, p. 045115, 2004.

[21] F. Natali, B. J. Ruck, N. O. V. Plank, H. J. Trodahl, S. Granville, C. Meyer, and W. R. L. Lambrecht, "Rare-earth mononitrides", Prog. Mats. Sci., vol. 58, no. 132409, 2013.

[22] G. Busch, "Magnetic properties of rare-earth compounds", J. Appl. Phys., vol. 38, no. 1386, 1967.

[23] R. A. Cutler and A. W. Lawson, "Synthesis and magnetic behavior of GdN", J. Appl. Phys., vol. 46, no. 2739, 1975.

[24] F. Natali, S. Vezian, S. Granville, B. Damilano, H. J. Trodahl, E.-M. Anton, H. Warring, F. Semond, Y. Cordier, S. V. Chong, and B. J. Ruck, "Molecular beam epitaxy of ferromagnetic epitaxial GdN thin films", J. Cryst. Growth, vol. 404, no. 146, 2014.

[25] B. M. Ludbrook, I. L. Farrell, M. Kuebel, B. J. Ruck, A. R. H. Preston, H. J. Trodahl, L. Ranno, R. J. Reeves, and S. M. Durbin, "Growth and properties of epitaxial GdN", J. Appl. Phys., vol. 106, no. 063910, 2009.

[26] S. Cwik, S. M. J. Beer, S. Hoffmann, M. Krasnopolski, D. Rogalla, H.W. Becker, D. Peeters, A. Ney, and A. Devi, "Integrating AlN with 
GdN Thin Films in an in Situ CVD Process: Influence on the Oxidation and Crystallinity of GdN", ACS Appl. Mater. Interfaces, vol. 9, pp. 27036-27044, 2017.

[27] C. M. Lee, H. Warring, S. Vézian, B. Damilano, S. Granville, M. Al Khalfioui, Y. Cordier, H. J. Trodahl, B. J. Ruck, and F. Natali, “Highly resistive epitaxial Mg-doped GdN thin films", Appl. Phys. Lett., vol. 106, p. 022401, 2015.

[28] F. Natali, B. J. Ruck, H. J. Trodahl, D. L. Binh, S. Vezian, B. Damilano, Y. Cordier, F. Semond, and C. Meyer, "Role of magnetic polarons in ferromagnetic GdN", Phys. Rev. B, vol. 87, no. 035202, 2013.

[29] F. Natali, B. Ludbrook, J. Galipaud, N. Plank, S. Granville, A. Preston, B. L. Do, J. Richter, I. Farrell, R. Reeves, S. Durbin, J. Trodahl, and B. Ruck, "Epitaxial growth and properties of GdN, EuN and SmN thin films", Phys. Status Solidi C, vol. 9, no. 605, 2012.

[30] K. Senapati, M. G. Blamire, and Z. H. Barber, "Spin-filter Josephson junctions", Nat. Mater., vol. 10, no. 849, 2011.

[31] H. Warring, B. J. Ruck, H. J. Trodahl, and F. Natali, “Electric field and photo-excited control of the carrier concentration in GdN", Appl. Phys. Lett., vol. 102, no. 132409, 2013.

[32] A. Pal, K. Senapati, Z. H. Barber, and M. G. Blamire, “Electric-fielddependent spin polarization in GdN spin filter tunnel junctions", Adv. Mater., vol. 25, pp. 5581-5585, 2013.

[33] H. Warring, H. J. Trodahl, N. O. Plank, F. Natali, S. Granville, and B. J. Ruck, "Magnetic Tunnel Junctions Incorporating a Near-ZeroMoment Ferromagnetic Semiconductor", Phys. Rev. Appl., vol. 6, p. 044002, 2016.

[34] F. Natali, B. Ruck, J. Trodahl, and S. Vezian “Doped rare earth nitride materials and devices comprising same", US20170022632A1, 2015. 
[35] F. Natali, B. Ruck, J. Trodahl, and S. Vezian "Magnetic materials and devices comprising rare earth nitrides", US20170018346A1, 2015.

[36] D. S. Holmes, A. L. Ripple, and M. A. Manheimer, "Energy-Efficient Superconducting Computing-Power Budgets and Requirements", IEEE Trans. Appl. Supercond., vol. 23, pp. 1701610-1701610, 2013.

[37] A. Andrae and P. Corcoran, "Emerging Trends in Electricity Consumption for Consumer ICT", 2013. [Online]. Available: https://wireless.kth.se/wpcontent/uploads/sites/19/2014/08/Emerging-Trends-inElectricity-Consumption-for-Consumer-ICT.pdf. [Accessed 201805-23]

[38] M. Avgerinou, P. Bertoldi, and L. Castellazzi, “Trends in Data Centre Energy Consumption under the European Code of Conduct for Data Centre Energy Efficiency", Energies, vol. 10, p. 1470, 2017.

[39] Hypres, "Hypres Product Webpage", [Online]. Available: https://www.hypres.com/products/ [Accessed 2018-05-23]

[40] D. Apalkov, B. Dieny, and J. M. Slaughter, "Magnetoresistive Random Access Memory", Proc. IEEE, vol. 104, pp. 1796-1830, 2016.

[41] N. Ashcroft and N. Mermin, Solid-State Physics. San Diego: Harcourt College Publishers, 1976.

[42] L. H. Allen, M. Y. Zhang, J. W. Mayer, E. G. Colgan, and R. Young, "Solutions to current crowding in circular vias for contact resistance measurements", J. Appl. Phys., vol. 70, pp. 253-258, 1991.

[43] Dieter K. Schroder, Semiconductor Material and Device Characterization. Hoboken, NJ, USA: Wiley, 2006.

[44] S. Sze and K. K. Ng, Physics of Semiconductor Devices. Hoboken, NJ, USA: John Wiley \& Sons, Inc., 2006. 
[45] R. Holm, "The electric tunnel effect across thin insulator films in contacts", J. Appl. Phys., vol. 22, pp. 569-574, 1951.

[46] J. Frenkel, "On the Electrical Resistance of Contacts between Solid Conductors", Phys. Rev., vol. 36, pp. 1604-1618, 1930.

[47] S. Granville, B. J. Ruck, F. Budde, A. Koo, D. J. Pringle, F. Kuchler, A. R. H. Preston, D. H. Housden, N. Lund, A. Bittar, G. V. M. Williams, and H. J. Trodahl, "Semiconducting ground state of GdN thin films", Phys. Rev. B - Condens. Matter Mater. Phys., vol. 73, p. 235335, 2006.

[48] N. O. V. Plank, F. Natali, J. Galipaud, J. H. Richter, M. Simpson, H. J. Trodahl, and B. J. Ruck, "Enhanced Curie temperature in N-deficient GdN", Appl. Phys. Lett., vol. 98, no. 112503, pp. 6-8, 2011.

[49] F. Ullstad, J. R. Chan, H. Warring, N. Plank, B. Ruck, and J. Trodahl, "Ohmic contacts of $\mathrm{Au}$ and Ag metals to n-type GdN thin films", AIMS Materials Science, vol. 2, no. 2, pp. 79-85, 2015.

[50] M. G. Blamire, A. Pal, Z. H. Barber, and K. Senapati, "Spin filter superconducting tunnel junctions", Proc. SPIE, vol. 8461, p. 84610J, 2012.

[51] R. Meservey and P. M. Tedrow, "Spin-polarized electron tunneling", Physics reports, vol. 238, 1994. 